\title{
Data report: modal sand composition at Sites C0004, C0006, C0007, and C0008, IODP Expedition 316, Nankai accretionary prism ${ }^{1}$
}

\author{
Kitty L. Milliken, ${ }^{2}$ Emily E. Comer, ${ }^{3}$ and Kathleen M. Marsaglia ${ }^{4}$
}

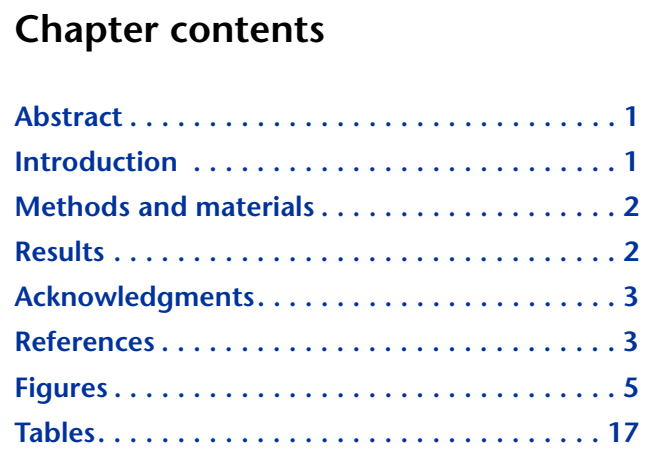

${ }^{1}$ Milliken, K.L., Comer, E.E., and Marsaglia, K.M., 2012. Data report: modal sand composition at Sites C0004, C0006, C0007, and C0008, IODP Expedition 316, Nankai accretionary prism. In Kinoshita, M., Tobin, H., Ashi, J., Kimura, G., Lallemant, S., Screaton, E.J., Curewitz, D., Masago, H., Moe, K.T., and the Expedition 314/315/316 Scientists, Proc. IODP, 314/315/316: Washington, DC (Integrated Ocean Drilling Program

Management International, Inc.). doi:10.2204/iodp.proc.314315316.221.2012 2Bureau of Economic Geology, Jackson School of Geosciences, University of Texas at Austin, Austin TX 78713, USA. kittym@mail.utexas.edu ${ }^{3}$ Department of Geosciences, College of Earth and Mineral Sciences, The Pennsylvania State University, University Park PA 16802, USA. ${ }^{4}$ Department of Geological Sciences, California State University Northridge, 18111 Nordhoff Street, Northridge CA 91330-8266, USA.

\section{Abstract}

Sand samples collected from cores recovered at Sites C0004, C0006, C0007, and C0008, Integrated Ocean Drilling Program Expedition 316 , are dominantly feldspathic litharenites containing between $18 \%$ and $40 \%$ detrital quartz within the total quartz, feldspar, lithic (QtFL) fraction. The lithic component includes a mix of volcanic, sedimentary, metamorphic, and plutonic fragments. The feldspar component is dominated by Ca-plagioclase. A grain-size control on modal composition is apparent, notably a greater concentration of feldspar grains in the finer samples. No significant stratigraphic or geographic trends in modal grain composition are observed within this sample set.

\section{Introduction}

Provenance of detrital grain populations is of interest for deciphering the tectonic and climatic influences on sediment supply during basin filling. In the case of accretionary prisms, provenance information has further ramifications for assessing the dynamic responses of prism growth and deformation to temporal variations of sediment generation and influx (e.g., Simpson, 2010).

Previous work on detrital modes in the Nankai forearc region has documented a wide range in composition that reflects variable mixing of volcanic, sedimentary, metasedimentary, and plutonic sources (Taira and Niitsuma, 1986; De Rosa et al., 1986; Marsaglia et al., 1992; Fergusson, 2003; Fergusson, 2011; Underwood and Fergusson, 2005).

Here we present a preliminary data set reporting on the modal grain compositions of 35 sand samples ( $>62 \mu \mathrm{m}$ fraction) collected at Sites C0004, C0006, C0007, and C0008 during Integrated Ocean Drilling Program (IODP) Expedition 316 (Fig. F1) (see the "Expedition 316 summary" chapter [Screaton et al., 2009]) within the Nankai accretionary prism south of the Kii Peninsula. Data were initially reported by Comer (2008). Low geothermal gradients along this segment of the forearc ensures that thermally driven diagenesis is minimal; burial temperatures are $<20^{\circ} \mathrm{C}$ for the deepest samples at all the sites reported here (see the "Expedition 316 summary" chapter [Screaton et al., 2009]). 


\section{Methods and materials}

Samples of unconsolidated sand $\left(10 \mathrm{~cm}^{3}\right.$ samples $)$ were collected during routine shipboard sampling with a sampling density of $\sim 1$ sample/core, limited by the availability of sand-size sediment. A total of 87 sand samples, 81 of Pleistocene age and 6 of Pliocene age, were obtained. A correlated set of mud samples, 166 Pleistocene, 53 Pliocene, and 3 Miocene, was also collected and used in a study by Milliken and Reed (2010). This report describes 35 sand samples distributed across the depth ranges available in the cores (Fig. F2). The time available for the senior honors project on which this report is based did not allow for a larger number of samples to be examined.

The fine fraction (detrital clay and silt) was removed from the samples by wet-sieving on a $62 \mu \mathrm{m}$ screen to isolate clean sand-size material. Sand was dried, made into grain mounts using blue-dyed epoxy, thin sectioned, and stained for identification of Ca-plagioclase and K-feldspar. Staining follows the procedure of Houghton (1980).

Thin sections were point-counted using the GazziDickinson method (Dickinson, 1970; Ingersoll et al., 1984). A total of 500 grains were counted using a Swift automatic point-counter in order to achieve "maximum grid spacing to cover the entire slide" (Ingersoll et al., 1984). A total of 22 grain categories were chosen based on categorizations established in previous studies (Marsaglia, 1992; Marsaglia et al., 1992; Fergusson, 2003). These categories include monocrystalline quartz and feldspars, volcanic lithic grains, metamorphic lithic grains, sedimentary lithic grains, and accessory grains including biogenic grains, micas, and heavy minerals (Table T1). Local cementation of ash beds by carbonate minerals is reported in this volume (see the "Expedition 316 Site C0004," "Expedition 316 Site C0006," "Expedition 316 Site C0007," and "Expedition 316 Site C0008" chapters [Expedition 316 Scientists, 2009a, 2009b, 2009c, 2009d]), but cementation, grain replacement, or grain dissolution evident by transmitted light microscopy is very minor in the samples described here. No authigenic components evident by transmitted light microscopy are included among the point-count categories. Ternary plots are made using the spreadsheet of Zahid and Barbeau (2011).

Grain size estimation $(\phi)$ was made by comparison of thin sections to a visual comparator ( $1 / 4 \phi$ intervals) using a light table and a hand lens.

\section{Results}

Using the sandstone classification of Folk (1980), most of the samples are feldspathic litharenite, with the remainder being lithic arkose (Table T2; Fig. F3). All samples contain between $18 \%$ and $40 \%$ detrital quartz within the total quartz, feldspar, lithic (QtFL; normalized percentages of total quartz, feldspar, and lithic grains) grain population. The feldspar assemblage is strongly dominated by Ca-plagioclase (Fig. F4). Lithic fragments include a mix of sedimentary, metamorphic, and volcanic fragments (Fig. F5). Sedimentary lithics dominate over the other types in most samples. Sedimentary clasts include chert and siliciclastic lithologies with sand/silt/detrital clay ratios ranging from sandstone to mudstone (Fig. F6). Metamorphic clasts are dominantly pelitic in composition and include grains that display a range of crystal size, ranging from slate to schist (Fig. F7). Volcanic lithic clasts are intermediate to mafic in composition and include grains with felsitic, microlitic, and lathwork fabrics and also vitric grains (Fig. F8). Although not included within the point-count categories because of their generally coarse crystal size $(>62 \mu \mathrm{m})$, grains of plutonic origin are present (Fig. F9). Biogenic grains include fragments of foraminifers, radiolarians, diatoms, sponge spicules, and wood. Minor authigenic components observed during point counting include pyrite framboids and a few instances of grain aggregates cemented by gypsum. The gypsum engulfs sand grains but is highly displasive and in many instances almost devoid of included grains (Fig. F10); it is possibly a product of postcoring oxidation of the sulfide.

Despite use of the Gazzi-Dickinson point-count method, which is intended to normalize grain compositions across variations in grain size, our data display a correlation between grain size and detrital feldspar, which is more abundant in the finer sand samples (Fig. F11). This contrasts with the results of Marsaglia et al. (1992), who also used the GazziDickinson point-count method but found no such relationship. Furthermore, Expedition 316 sand compositions are somewhat more feldspathic than those reported by Marsaglia et al. (1992) for Deep Sea Drilling Project Sites 298, 582, and 583.

The polymict assemblages observed in the sand grain populations described here are similar those reported for gravels at Site C0007 (Fergusson, 2011). Pliocene samples at Site C0008 are slightly more lithic rich than Pleistocene samples at that site; however, the Pliocene samples at Site C0004 are slightly less lithic 
rich than the one Pleistocene sample. Thus, no clear trend with sample age can be established. Overall, compositions are more feldspathic than those reported by Marsaglia et al. (1992) for Sites 298, 582, and 583. Samples are less lithic rich than the accreted Quaternary trench-wedge samples at Ocean Drilling Program Site 1174 and less quartz rich than the accreted Pleistocene and Pliocene deposits beneath the slope-apron facies at Site 1175 as reported by Fergusson (2003).

\section{Acknowledgments}

Samples and/or data for this report were provided by the Integrated Ocean Drilling Program. This project was supported by postexpedition funding awarded to KLM from the Consortium for Ocean Leadership. Samples were provided by IODP. KLM also thanks the Expedition 316 Shipboard Scientific Party and especially the sedimentology team of Chris Fergusson, Uisdean Nicholson, and Michael Strasser for help with sampling. We thank Michael Underwood for a helpful review of the manuscript and for providing the stratigraphic columns used in Figure F2.

\section{References}

Comer, E., 2008. Modal analysis of sand from the Nankai accretionary prism [B.S. thesis]. Univ. Texas, Austin. http://www.lib.utexas.edu/geo/thesis_dissertations/ thesisabstracts $2 . h t m l \#$ comer

De Rosa, R., Zuffa, G.G., Taira, A., and Leggett, J.K., 1986. Petrography of trench sands from the Nankai Trough, southwest Japan: implications for long-distance turbidite transportation. Geol. Mag., 123(5):477-486. doi:10.1017/S0016756800035068

Dickinson, W.R., 1970. Interpreting detrital modes of graywacke and arkose. J. Sediment. Res., 40(2):695-707. doi:10.1306/74D72018-2B21-11D78648000102C1865D

Expedition 316 Scientists, 2009a. Expedition 316 Site C0004. In Kinoshita, M., Tobin, H., Ashi, J., Kimura, G., Lallemant, S., Screaton, E.J., Curewitz, D., Masago, H., Moe, K.T., and the Expedition 314/315/316 Scientists, Proc. IODP, 314/315/316: Washington, DC (Integrated Ocean Drilling Program Management International, Inc.). doi:10.2204/iodp.proc.314315316.133.2009

Expedition 316 Scientists, 2009b. Expedition 316 Site C0006. In Kinoshita, M., Tobin, H., Ashi, J., Kimura, G., Lallemant, S., Screaton, E.J., Curewitz, D., Masago, H., Moe, K.T., and the Expedition 314/315/316 Scientists, Proc. IODP, 314/315/316: Washington, DC (Integrated Ocean Drilling Program Management International, Inc.). doi:10.2204/iodp.proc.314315316.134.2009

Expedition 316 Scientists, 2009c. Expedition 316 Site C0007. In Kinoshita, M., Tobin, H., Ashi, J., Kimura, G., Lallemant, S., Screaton, E.J., Curewitz, D., Masago, H.,
Moe, K.T., and the Expedition 314/315/316 Scientists, Proc. IODP, 314/315/316: Washington, DC (Integrated Ocean Drilling Program Management International, Inc.). doi:10.2204/iodp.proc.314315316.135.2009

Expedition 316 Scientists, 2009d. Expedition 316 Site C0008. In Kinoshita, M., Tobin, H., Ashi, J., Kimura, G., Lallemant, S., Screaton, E.J., Curewitz, D., Masago, H., Moe, K.T., and the Expedition 314/315/316 Scientists, Proc. IODP, 314/315/316: Washington, DC (Integrated Ocean Drilling Program Management International, Inc.). doi:10.2204/iodp.proc.314315316.136.2009

Fergusson, C.L., 2003. Provenance of Miocene-Pleistocene turbidite sands and sandstones, Nankai Trough, Ocean Drilling Program Leg 190. In Mikada, H., Moore, G.F., Taira, A., Becker, K., Moore, J.C., and Klaus, A. (Eds.), Proc. ODP, Sci. Results, 190/196: College Station, TX (Ocean Drilling Program), 1-28. doi:10.2973/ odp.proc.sr.190196.205.2003

Fergusson, C.L., 2011. Data report: clast counts and petrography of gravels from Site C0007, IODP Expedition 316, Nankai Trough. In Kinoshita, M., Tobin, H., Ashi, J., Kimura, G., Lallemant, S., Screaton, E.J., Curewitz, D., Masago, H., Moe, K.T., and the Expedition 314/315/316 Scientists, Proc. IODP, 314/315/316: Washington, DC (Integrated Ocean Drilling Program Management International, Inc.). doi:10.2204/ iodp.proc.314315316.203.2011

Folk, R.L., 1980. Petrology of Sedimentary Rocks (2nd ed.): Austin, TX (Hemphill's).

Houghton, H.F., 1980. Refined techniques for staining plagioclase and alkali feldspars in thin section. J. Sediment. Res., 50(2):629-631. http://jsedres.geoscienceworld.org/content/50/2/629.abstract

Ingersoll, R.V., Bullard, T.F., Ford, R.L., Grimm, J.P., Pickle, J.D., and Sares, S.W., 1984. The effect of grain size on detrital modes: a test of the Gazzi-Dickinson pointcounting method. J. Sediment. Res., 54(1):103-116. doi:10.1306/212F83B9-2B24-11D78648000102C1865D

Marsaglia, K.M., 1992. Petrography and provenance of volcaniclastic sands recovered from the Izu-Bonin arc, Leg 126. In Taylor, B., Fujioka, K., et al., Proc. ODP, Sci. Results, 126: College Station, TX (Ocean Drilling Program), 139-154. doi:10.2973/ odp.proc.sr.126.124.1992

Marsaglia, K.M., Ingersoll, R.V., and Packer, B.M., 1992. Tectonic evolution of the Japanese Islands as reflected in modal compositions of Cenozoic forearc and backarc sand and sandstone. Tectonics, 11(5):1028-1044. doi:10.1029/91TC03183

Milliken, K.L., and Reed, R.M., 2010. Multiple causes of diagenetic fabric anisotropy in weakly consolidated mud, Nankai accretionary prism, IODP Expedition 316. J. Struct. Geol., 32(12):1887-1898. doi:10.1016/ j.jsg.2010.03.008

Screaton, E.J., Kimura, G., Curewitz, D., and the Expedition 316 Scientists, 2009. Expedition 316 summary. In Kinoshita, M., Tobin, H., Ashi, J., Kimura, G., Lallemant, S., Screaton, E.J., Curewitz, D., Masago, H., Moe, K.T., and the Expedition 314/315/316 Scientists, Proc. 
IODP, 314/315/316: Washington, DC (Integrated Ocean Drilling Program Management International, Inc.). doi:10.2204/iodp.proc.314315316.131.2009

Simpson, G.D.H., 2010. Formation of accretionary prisms influenced by sediment subduction and supplied by sediments from adjacent continents. Geology, 38(2):131-134. doi:10.1130/G30461.1

Taira, A., and Niitsuma, N., 1986. Turbidite sedimentation in the Nankai Trough as interpreted from magnetic fabric, grain size, and detrital modal analyses. In Kagami, H., Karig, D.E., Coulbourn, W.T., et al., Init. Repts. DSDP, 87: Washington, DC (U.S. Govt. Printing Office), 611632. doi:10.2973/dsdp.proc.87.112.1986

Underwood, M.B., and Fergusson, C.L., 2005. Late Cenozoic evolution of the Nankai trench-slope system: Evi- dence from sand petrography and clay mineralogy. In Hodgson, D., and Flint, S. (Eds.), Submarine Slope Systems: Processes, Products and Prediction. Geol. Soc. Spec. Publ., 244(1):113-129. doi:10.1144/

GSL.SP.2005.244.01.07

Zahid, K.M., and Barbeau, D.L., Jr., 2011. Constructing sandstone provenance and classification ternary diagrams using an electronic spreadsheet. J. Sediment. Res., 81(9):702-707. doi:10.2110/jsr.2011.55

Initial receipt: 26 November 2011

Acceptance: 2 February 2012

Publication: 6 April 2012

MS 314315316-221 
Figure F1. Map showing locations of Sites C0004, C0008, C0006, and C0007. Sites for which modal sand composition data are available from the previous studies cited in Figure F3 are also indicated. Modified from Screaton et al. (2009). KPR = Kyushu-Palau Ridge, FSC = fossil spreading center, PSP = Philippine Sea plate, IBT = Izu-Bonin Trench.

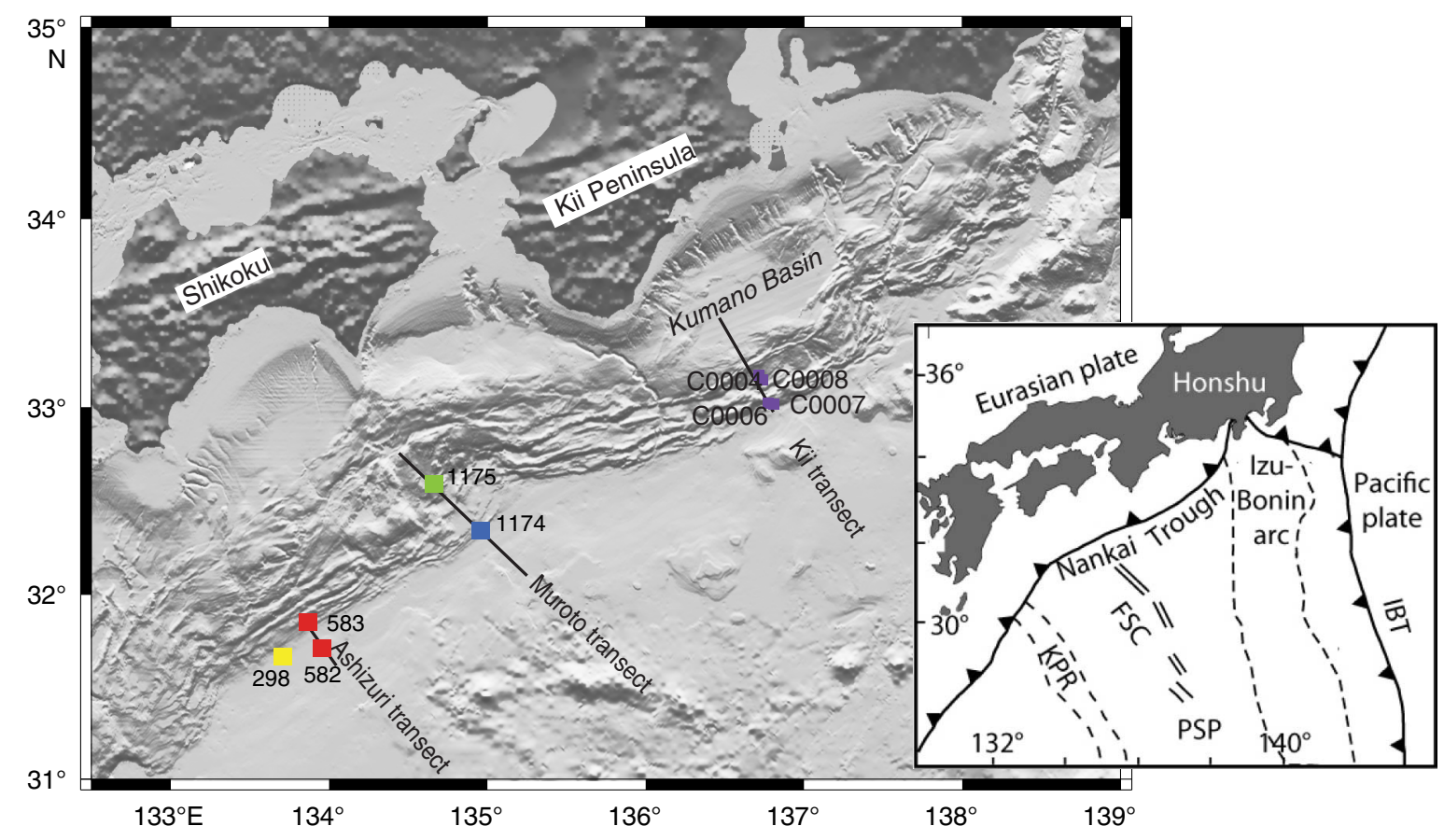


Figure F2. Distribution of sample set with respect to lithostratigraphy. Sample depths (in mbsf) are indicated on the left side of each graphical core log. A. Frontal thrust cores, Sites C0004 and C0008. (Figure continued on next page.)

A

Megasplay fault zone

\section{Site $\mathbf{C 0 0 0 8}$}

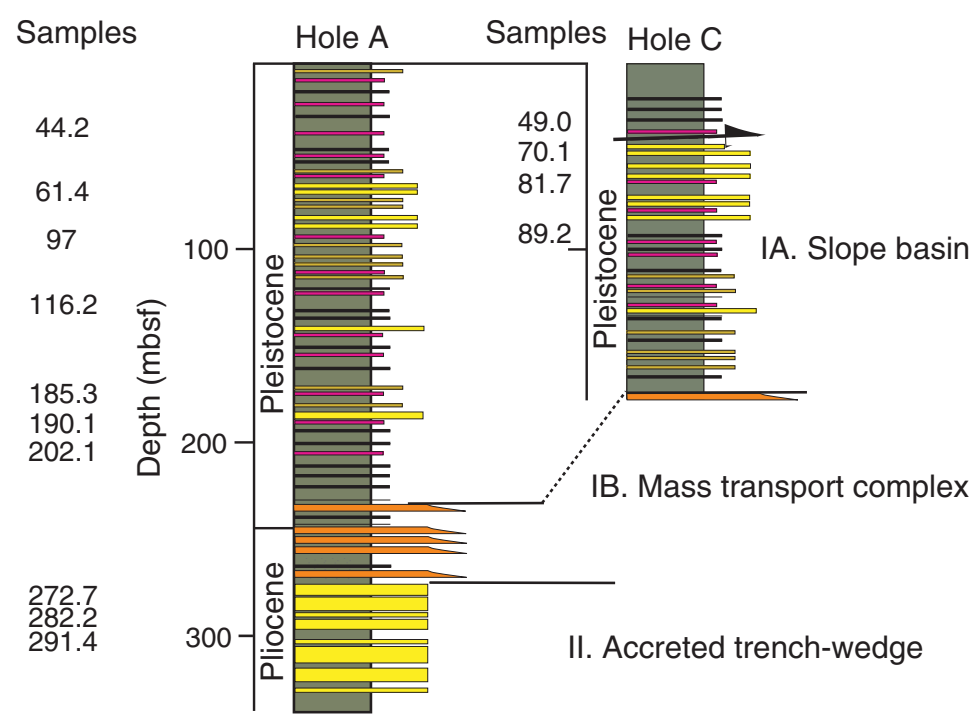

Site $\mathrm{C0004}$

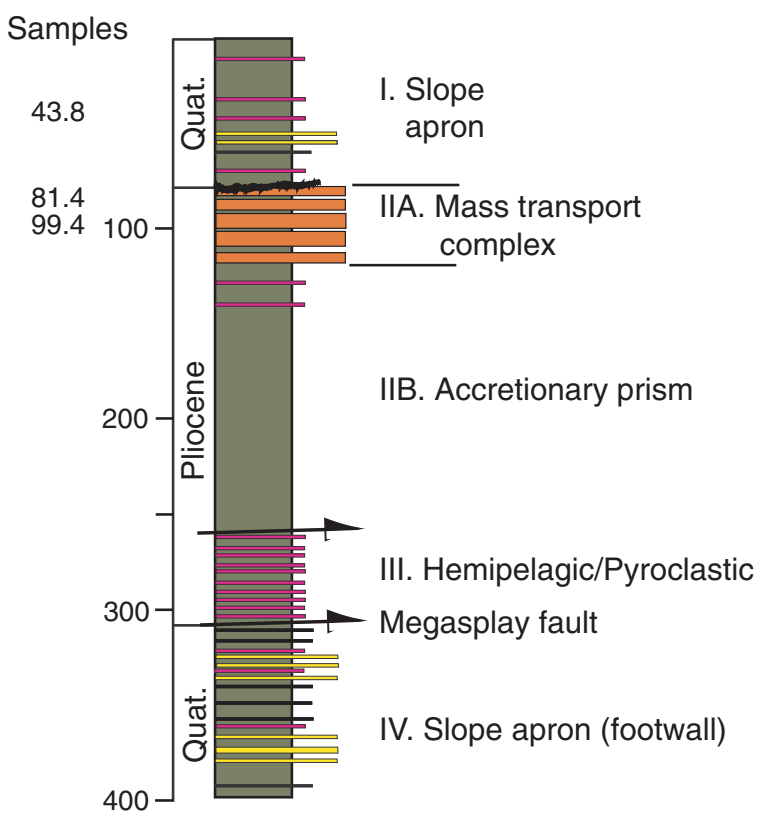

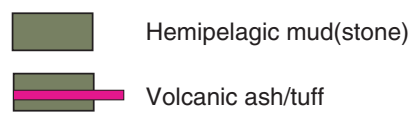
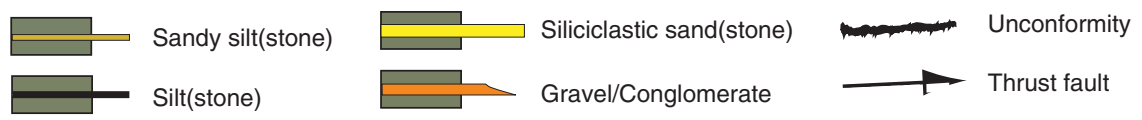
Figure F2 (continued). B. Megasplay cores, Sites C0006 and C0007.

B

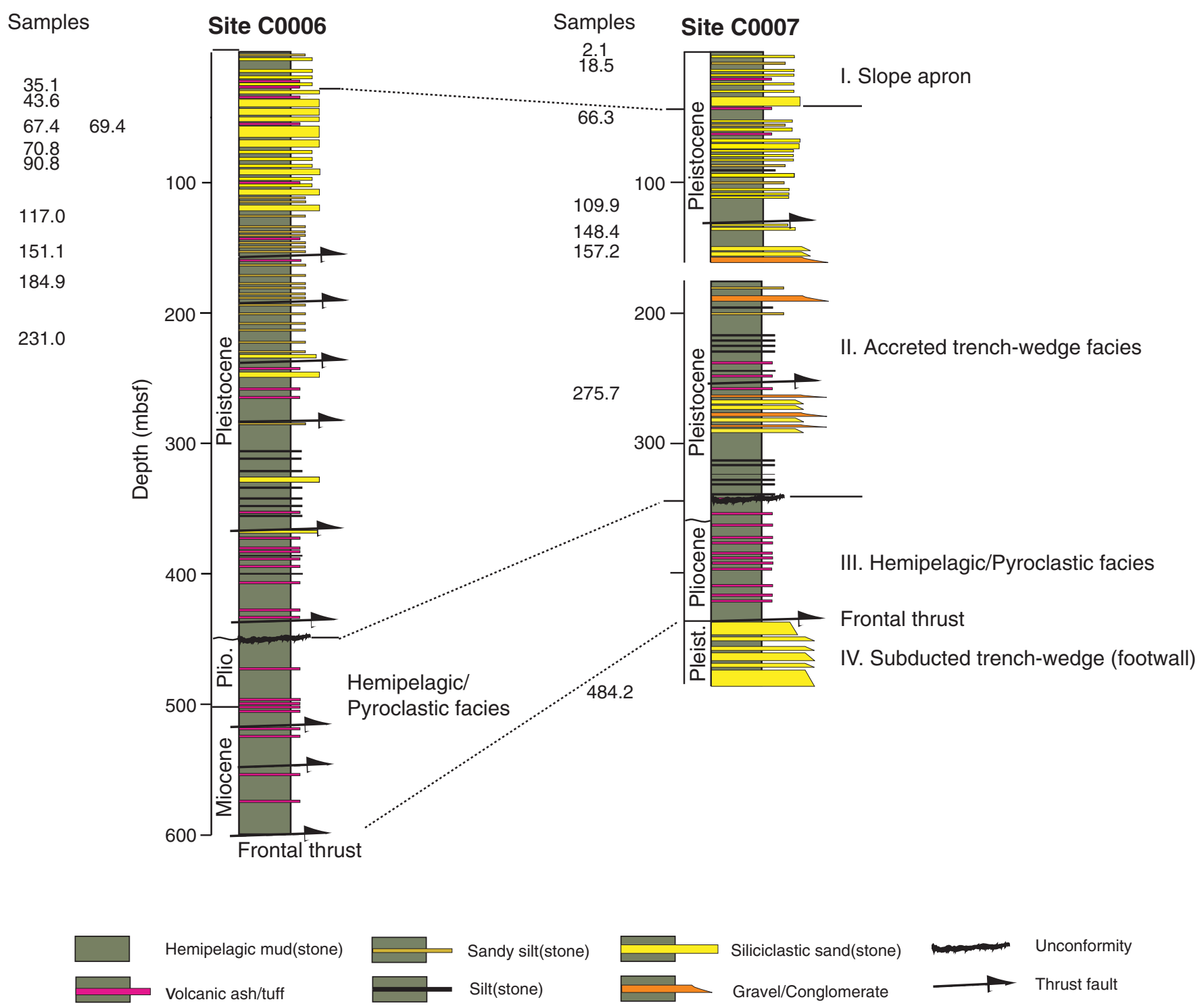

\section{Frontal thrust zone}


Figure F3. Major grain composition plots: $\mathrm{Qt}=$ total quartz, $\mathrm{F}=$ feldspar, $\mathrm{L}=$ lithic grains. Data from Marsaglia et al. (1992) are average values. Data from Fergusson (2003) are shown as fields. A. Sites C0006 and C0007. B. Sites C0004 and C0008.

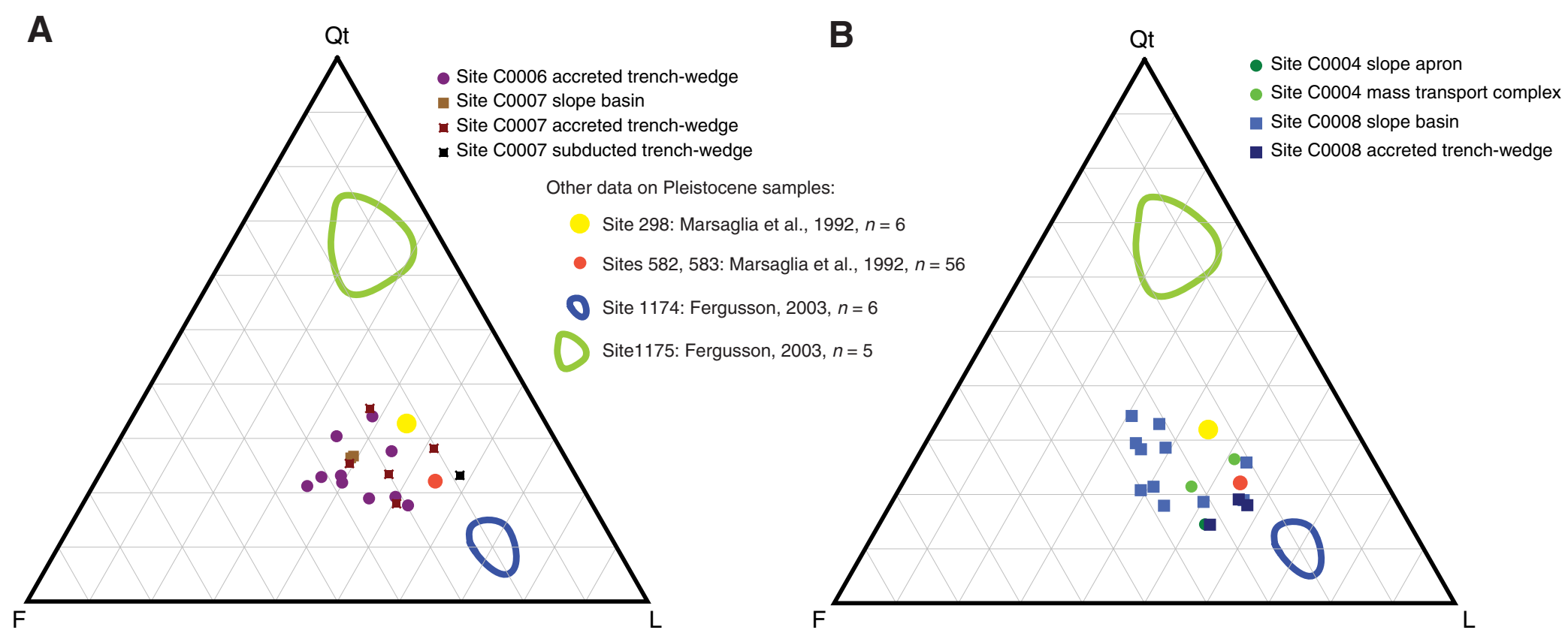


Figure F4. Monocrystalline quartz (Qm)-Ca-plagioclase (P)-K-feldspar (K) triangles. A. Sites C0006 and C0007. B. Sites C0004 and C0008.
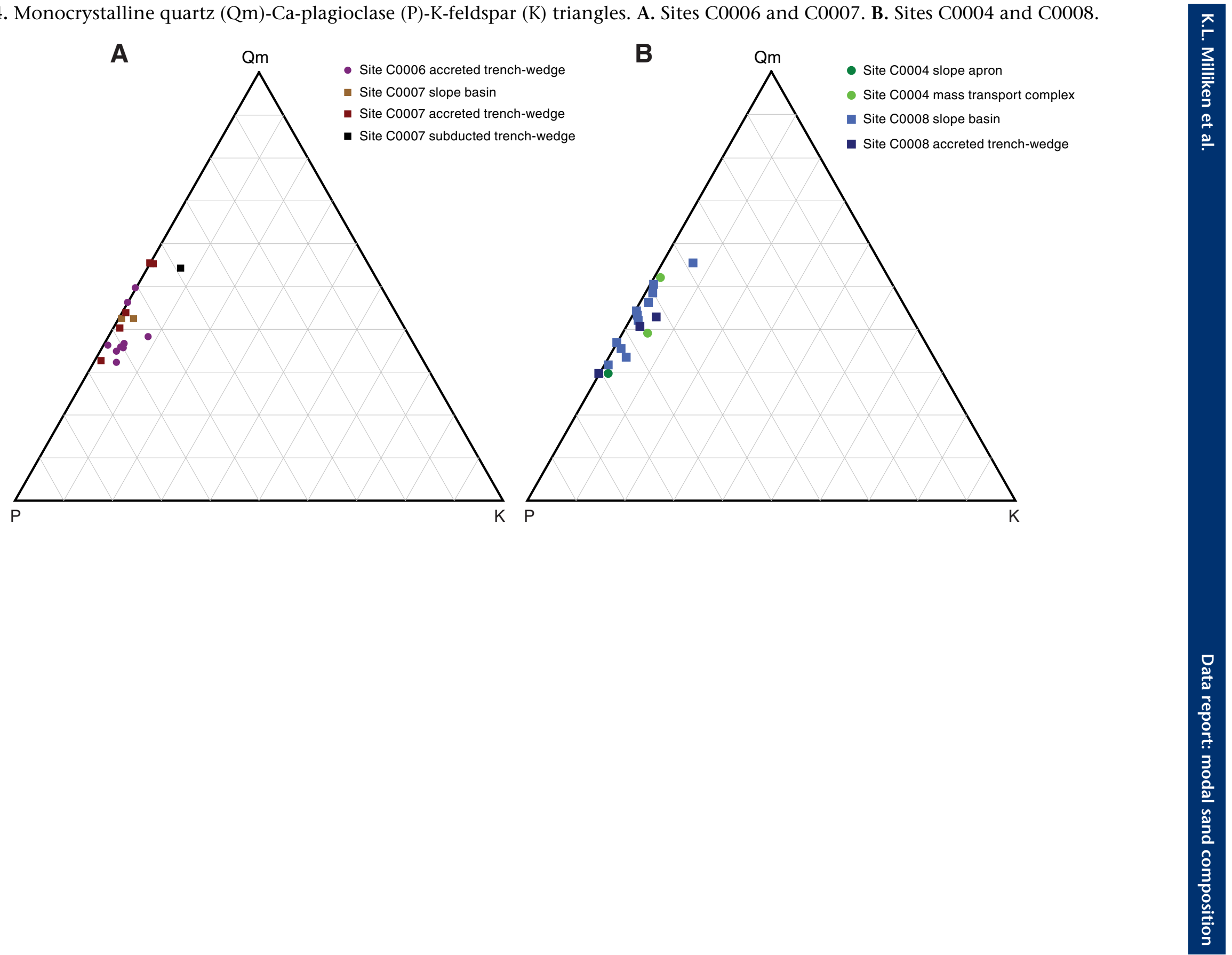
Figure F5. Composition of lithic fragment populations. Metamorphic lithic (rock) fragments (MRF)-volcanic lithic (rock) fragments (VRF)-sedimentary lithic (rock) fragments (SRF) triangle of Folk (1980). A. Sites C0006 and C0007. B. Sites C0004 and C0008.
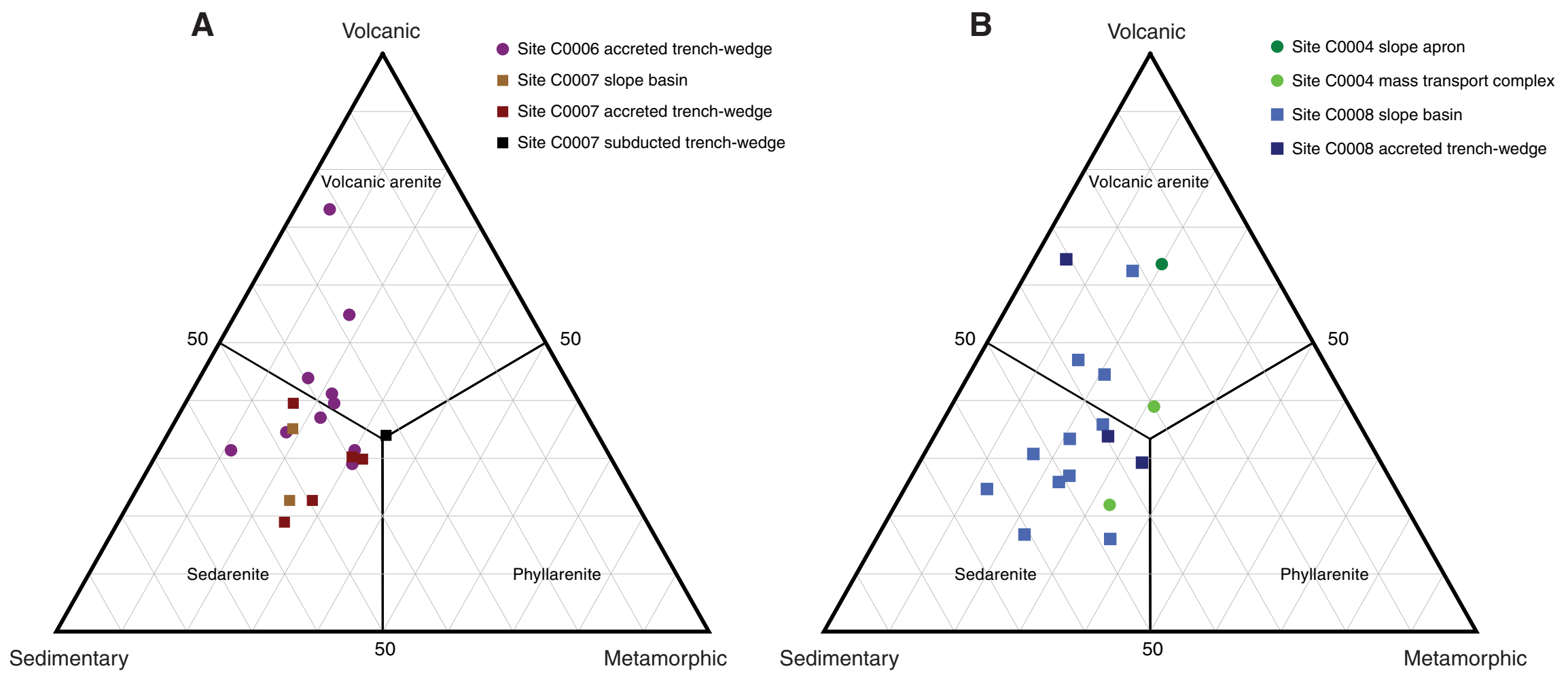
Figure F6. Sedimentary lithic clasts. Left-side images are taken in plane-polarized light. Right-side images are taken in cross-polarized light. A, B. Sandstone fragment (Sample 316-C0008A-35X-CC, 19-20 cm). C, D. Silty mudstone fragment (Sample 316-C0008A-35X-CC, 19-20 cm). E, F. Claystone fragment (Sample 316-C0008A34X-1, 44-45 cm). G, H. Chert (Sample 316-C0008A-34X-1, 44-45 cm).

A

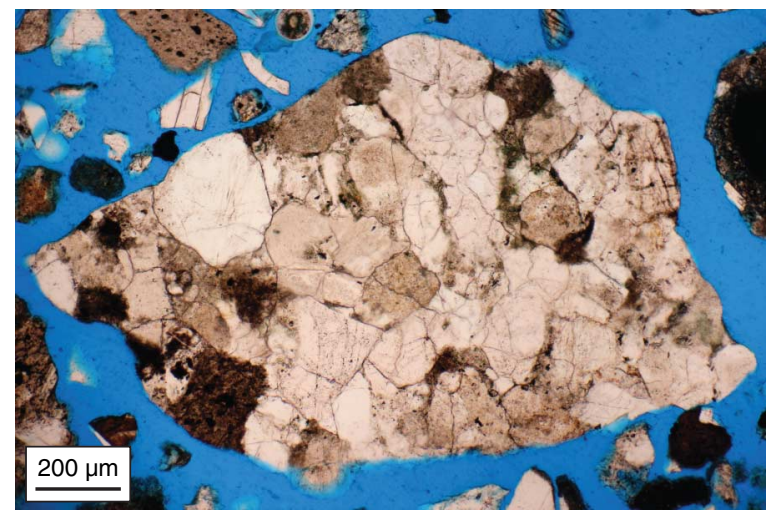

C

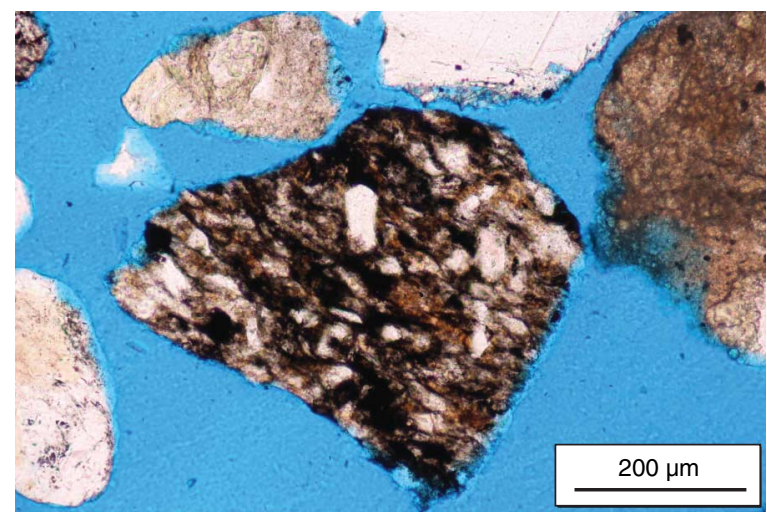

$\mathbf{E}$

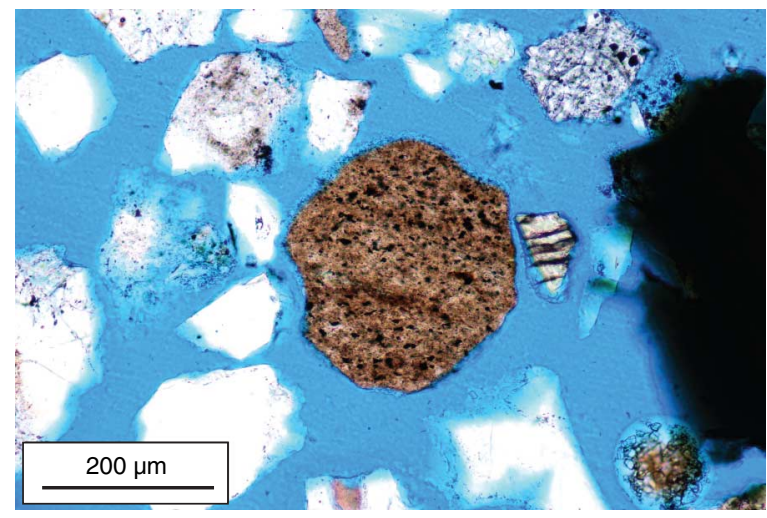

G

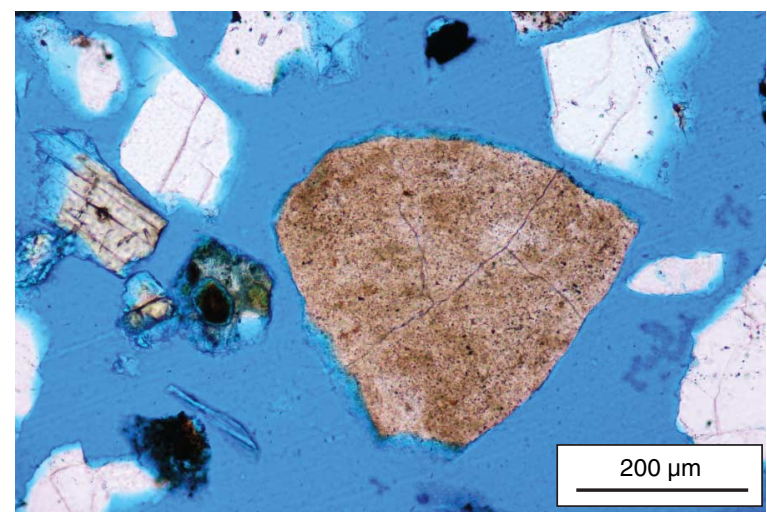

B

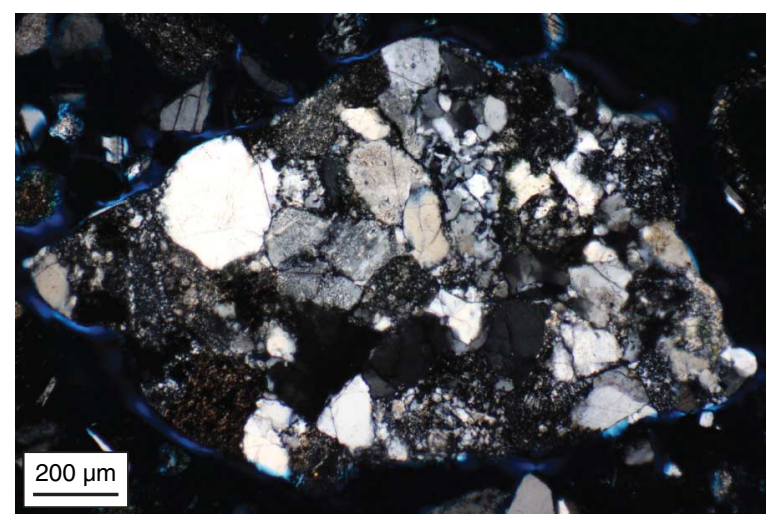

D

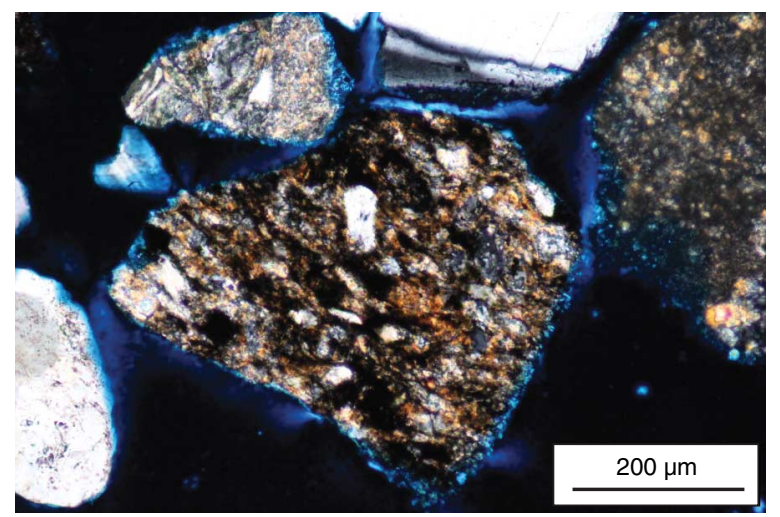

F

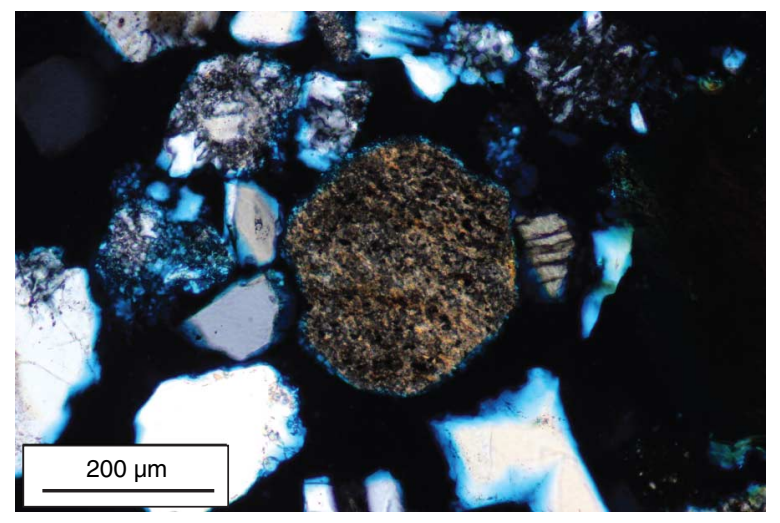

H

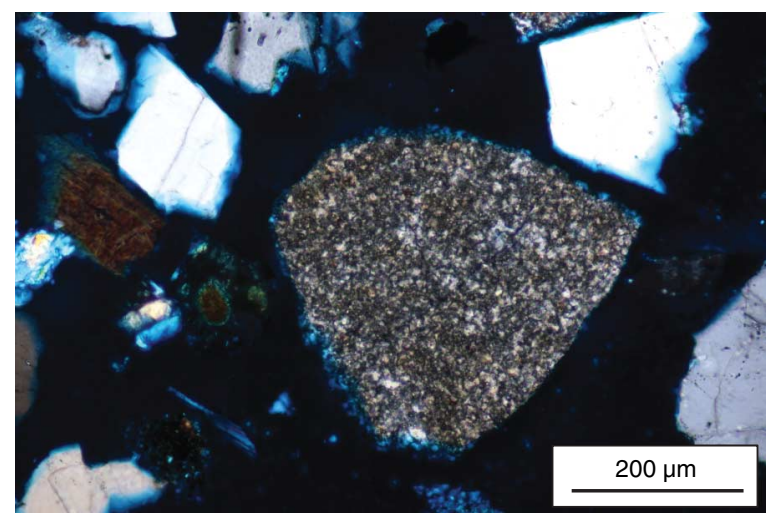


Figure F7. Metamorphic lithic clasts. Left-side images are taken in plane-polarized light. Right-side images are taken in cross-polarized light. A, B. Quartz-mica schist (Sample 316-C0007C-12X-1, 24-26 cm). C, D. QuartzCa-plagioclase schist (Sample 316-C0007C-12X-1, 24-26 cm). E, F. Quartz-mica phyllite (Sample 316-C0008A34X-1, 44-45 cm). G, H. Quartz-mica slate (Sample 316-C0007C-17H-1, 13-15 cm).

A

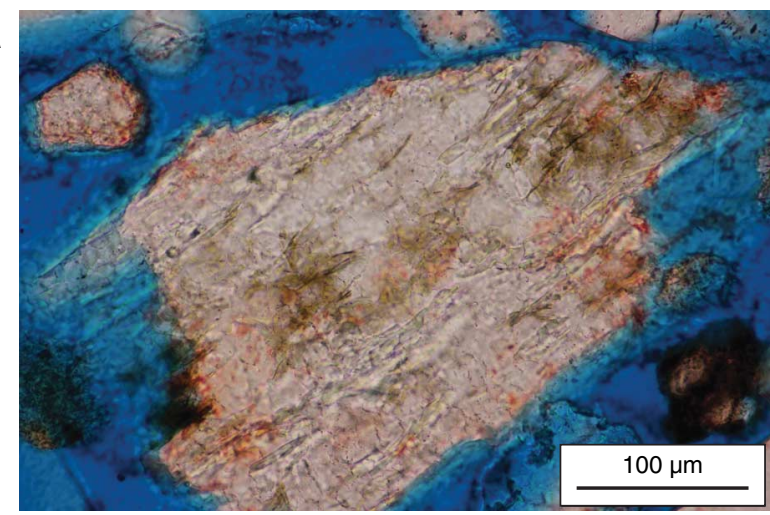

C

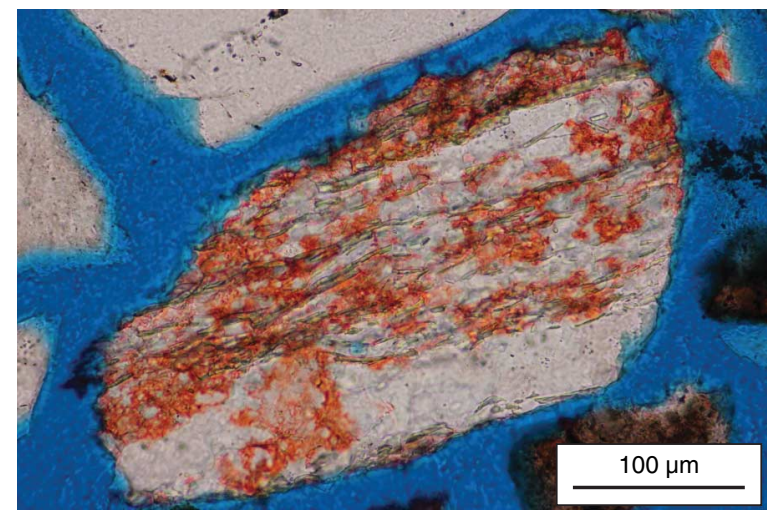

E

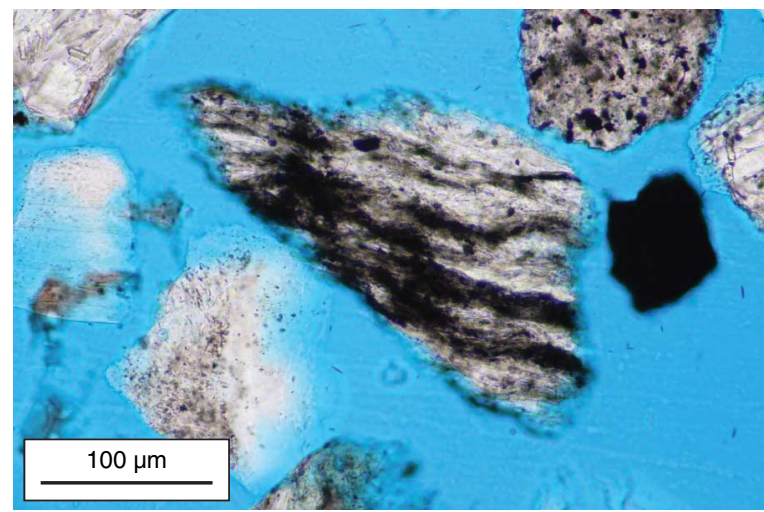

G

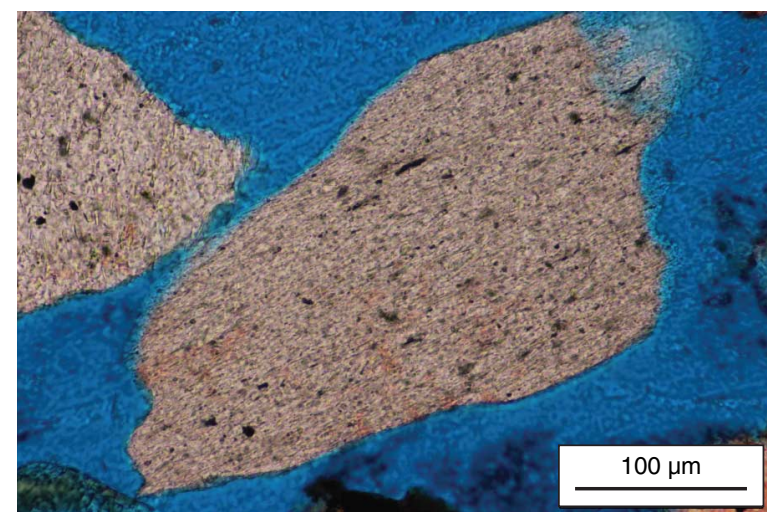

B

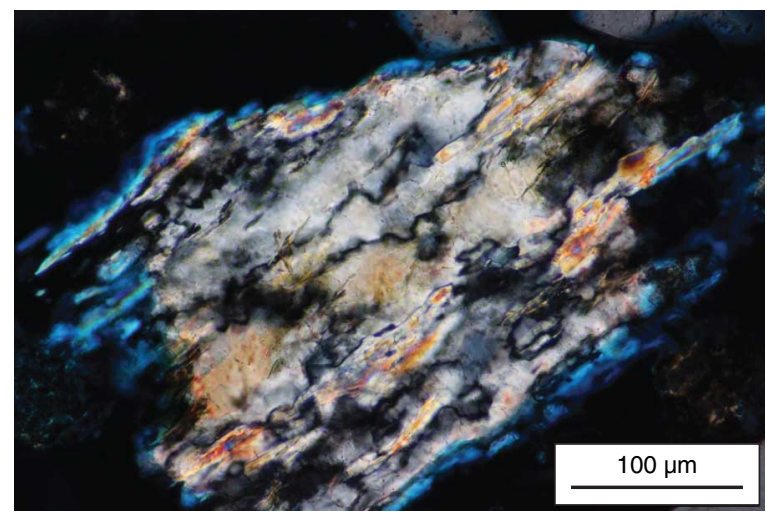

D

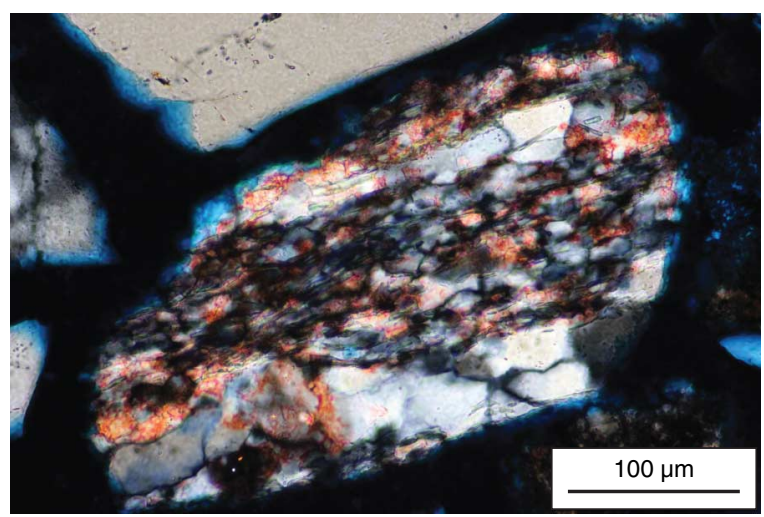

$\mathbf{F}$

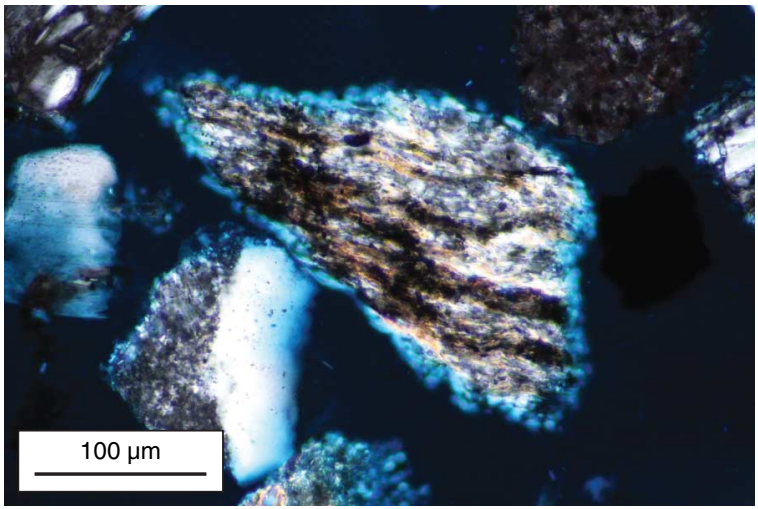

H

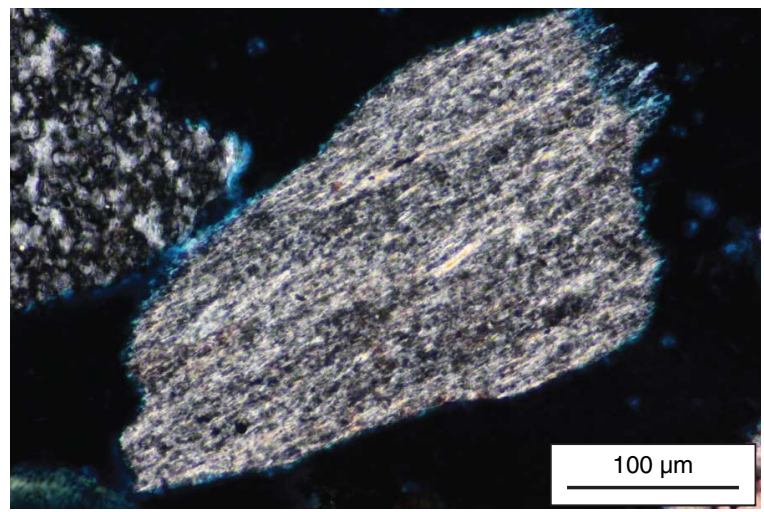


Figure F8. Volcanic lithic clasts. Except as noted, left-side images are taken in plane-polarized light and rightside images are taken in cross-polarized light. A. Simple glass (g) fragments (Sample 316-C0004C-5H-8, 18-20 $\mathrm{cm})$. B. Porous pumice fragment in plane-polarized light (Sample 316-C0004C-5H-8, 18-20 cm). C, D. Microlitic volcanic rock fragment (Sample 316-C0008A-43X-1, 44-45 cm). E, F. Lathwork volcanic rock fragment (Sample 316-C0008A-24H-3, 110-112 cm).
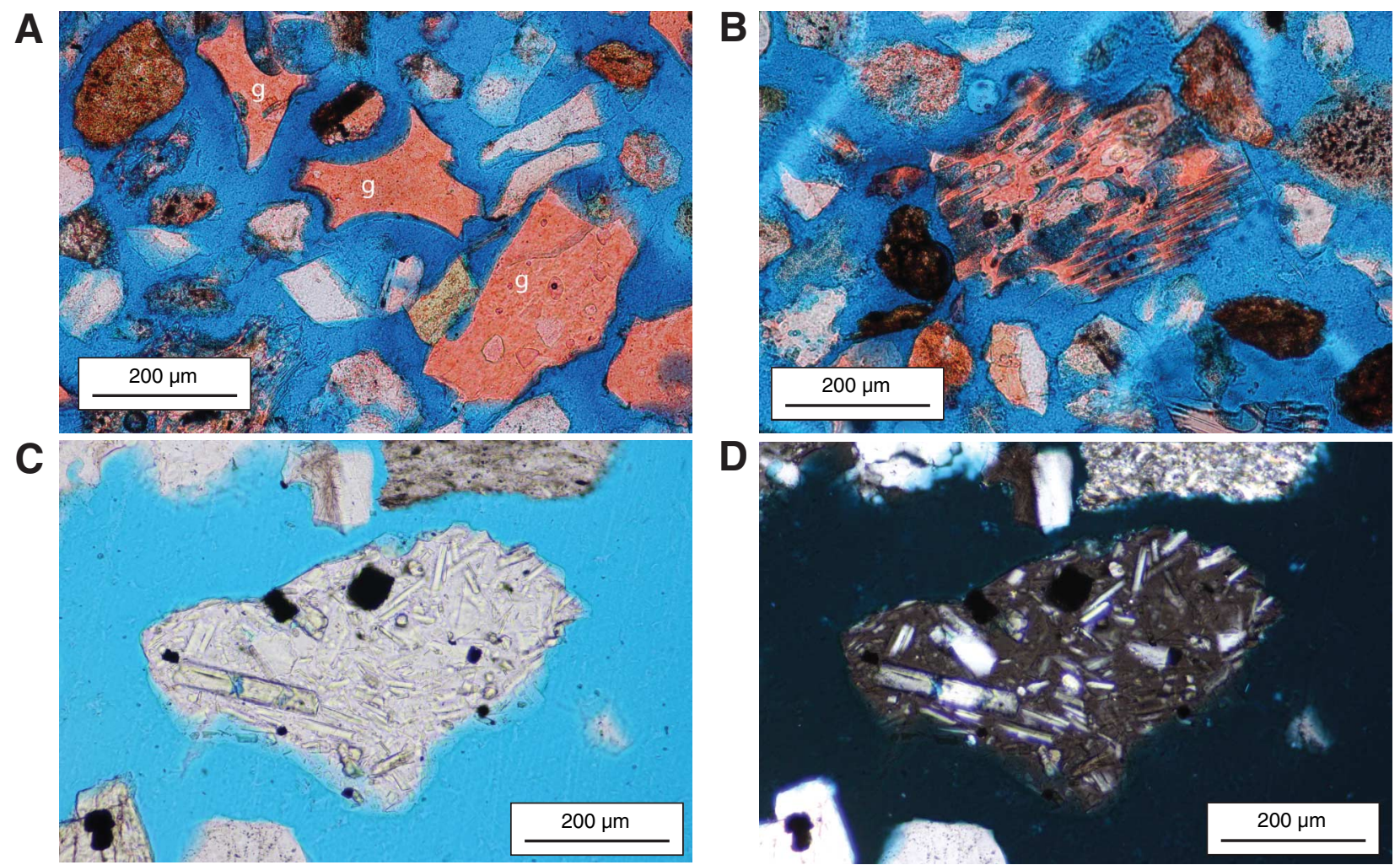

E
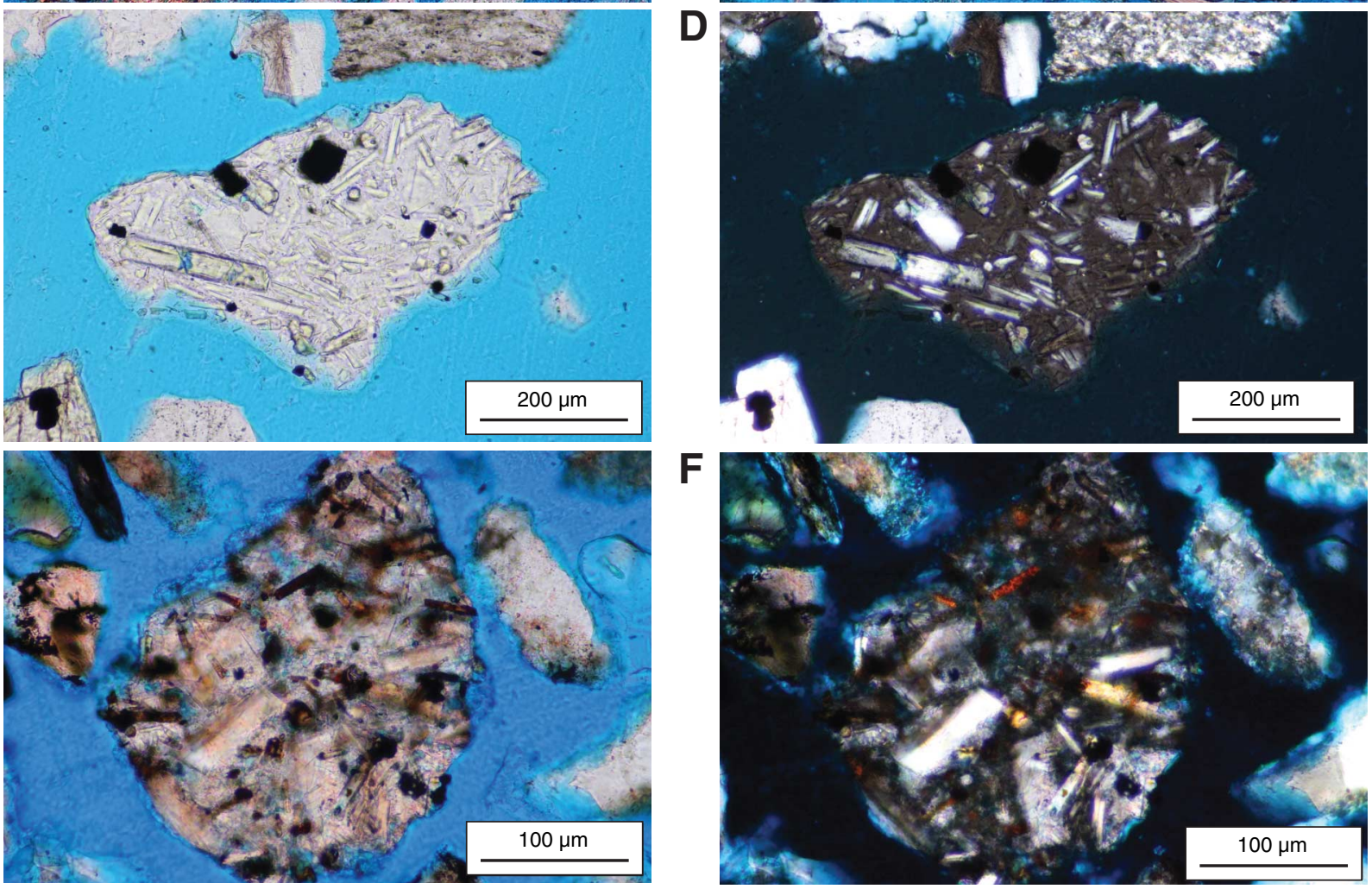

\section{$\mathbf{F}$}

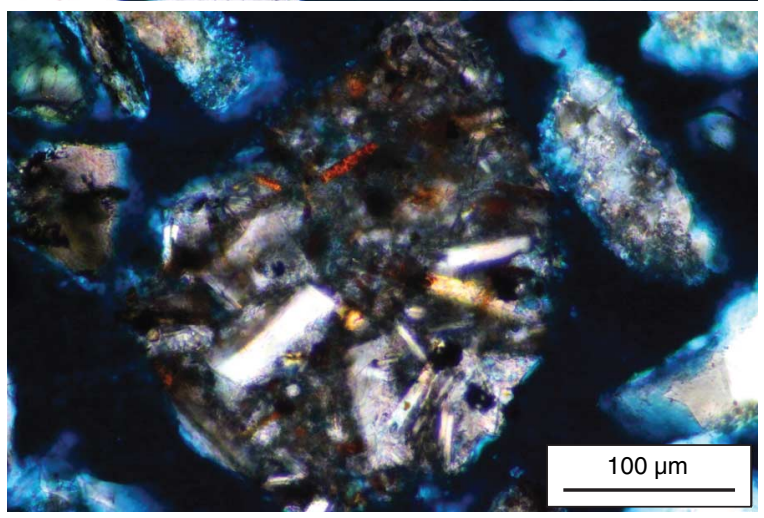


Figure F9. Plutonic lithic clasts (Sample 316-C0007C-12X-1, 24-26 cm). Left-side images are taken in planepolarized light and right-side images are taken in cross-polarized light. A, B. Quartz-Ca-plagioclase plutonic lithic. C, D. Mafic plutonic lithic (Ca-plagioclase + amphibole/pyroxene). E, F. Graphic feldspar/quartz intergrowth.
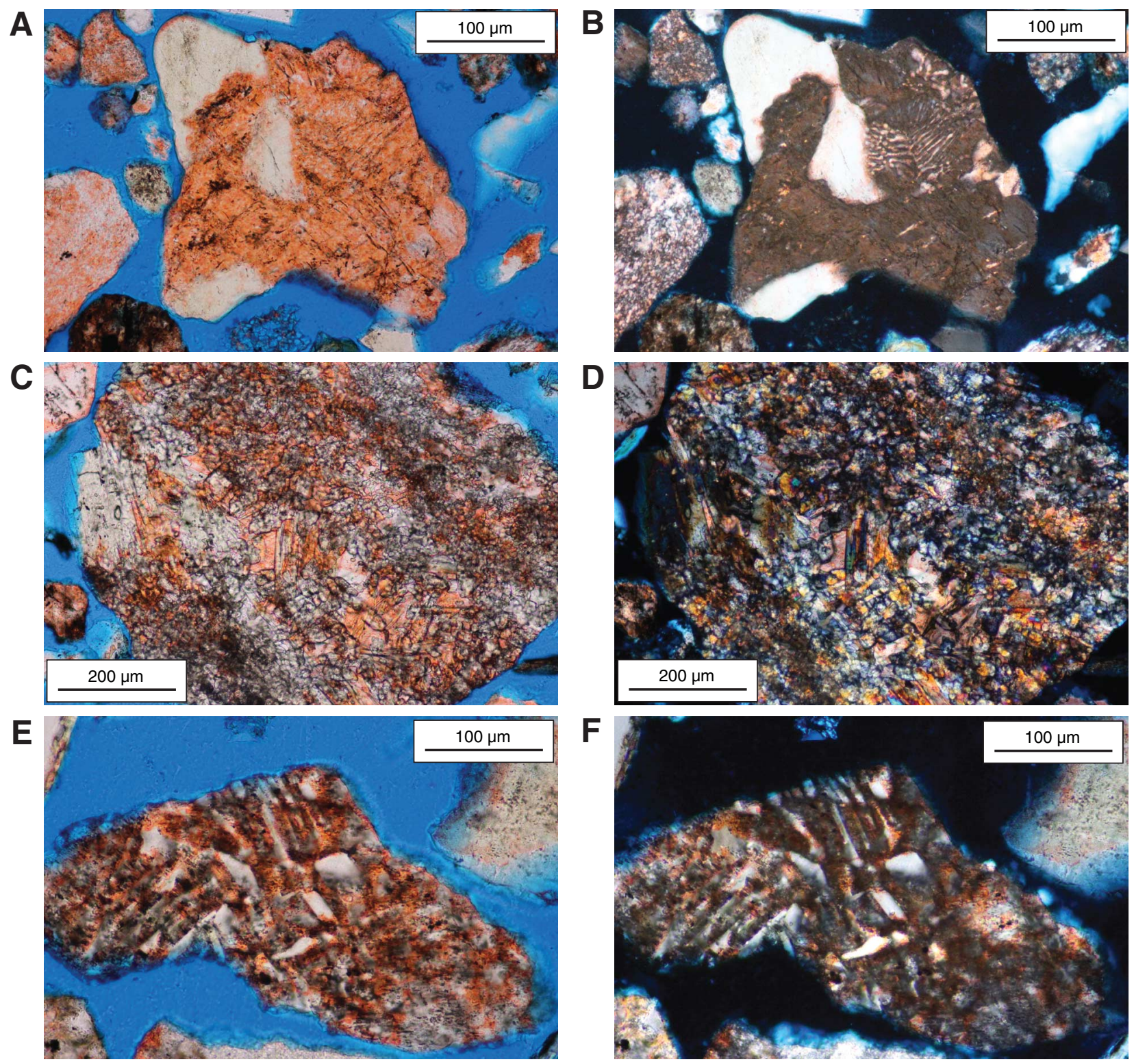
Figure F10. Small grain aggregates cemented by gypsum (Sample 316-C0006E-26X-2, 109-111 cm). Left-side images are taken in plane-polarized light and right-side images are taken in cross-polarized light. A, B. Grain aggregate cemented by gypsum. C, D. Gypsum spherule with minor detrital inclusions.
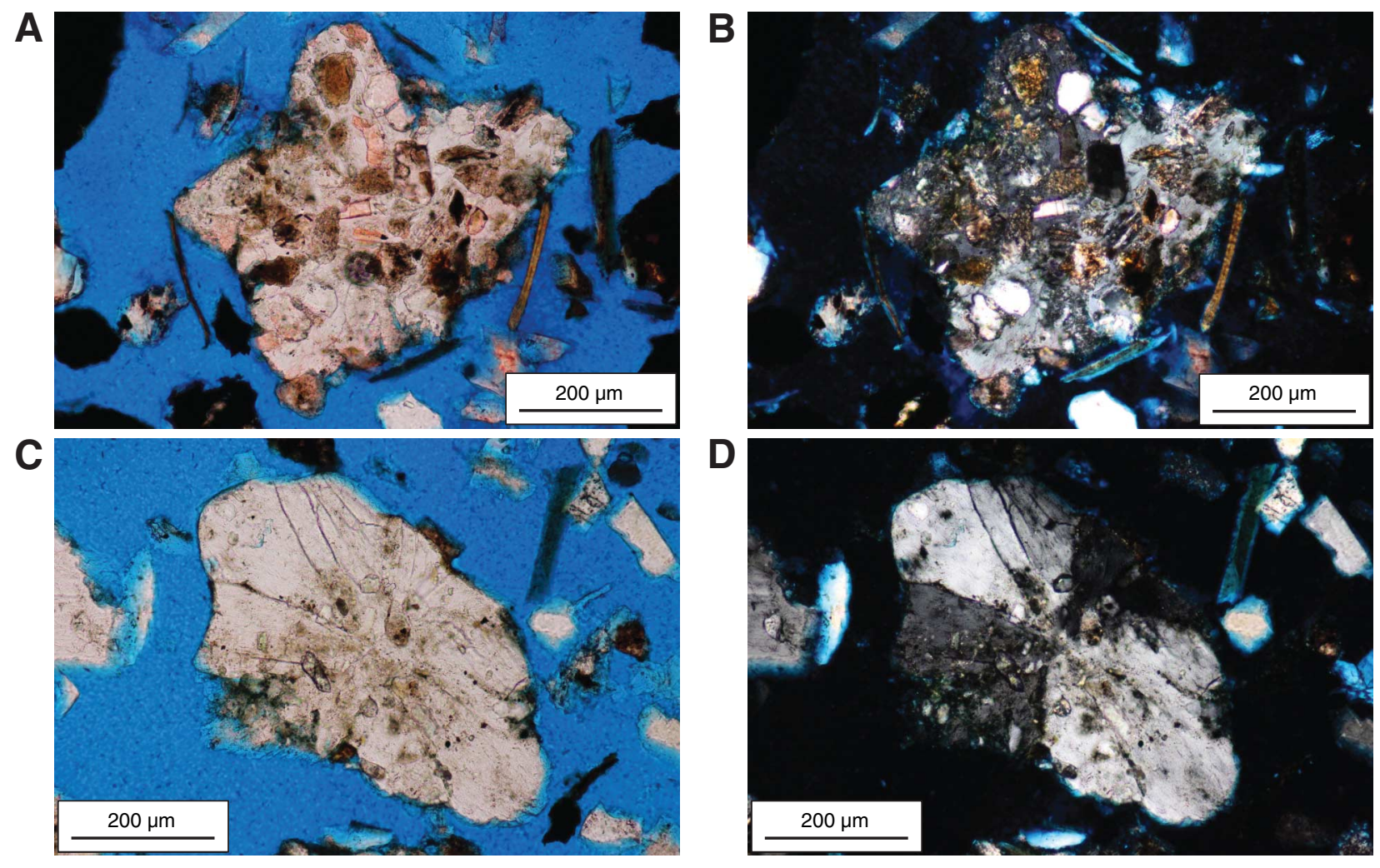
Figure F11. Detrital feldspar content versus grain size. QtFL = total quartz, feldspar, lithic fraction.

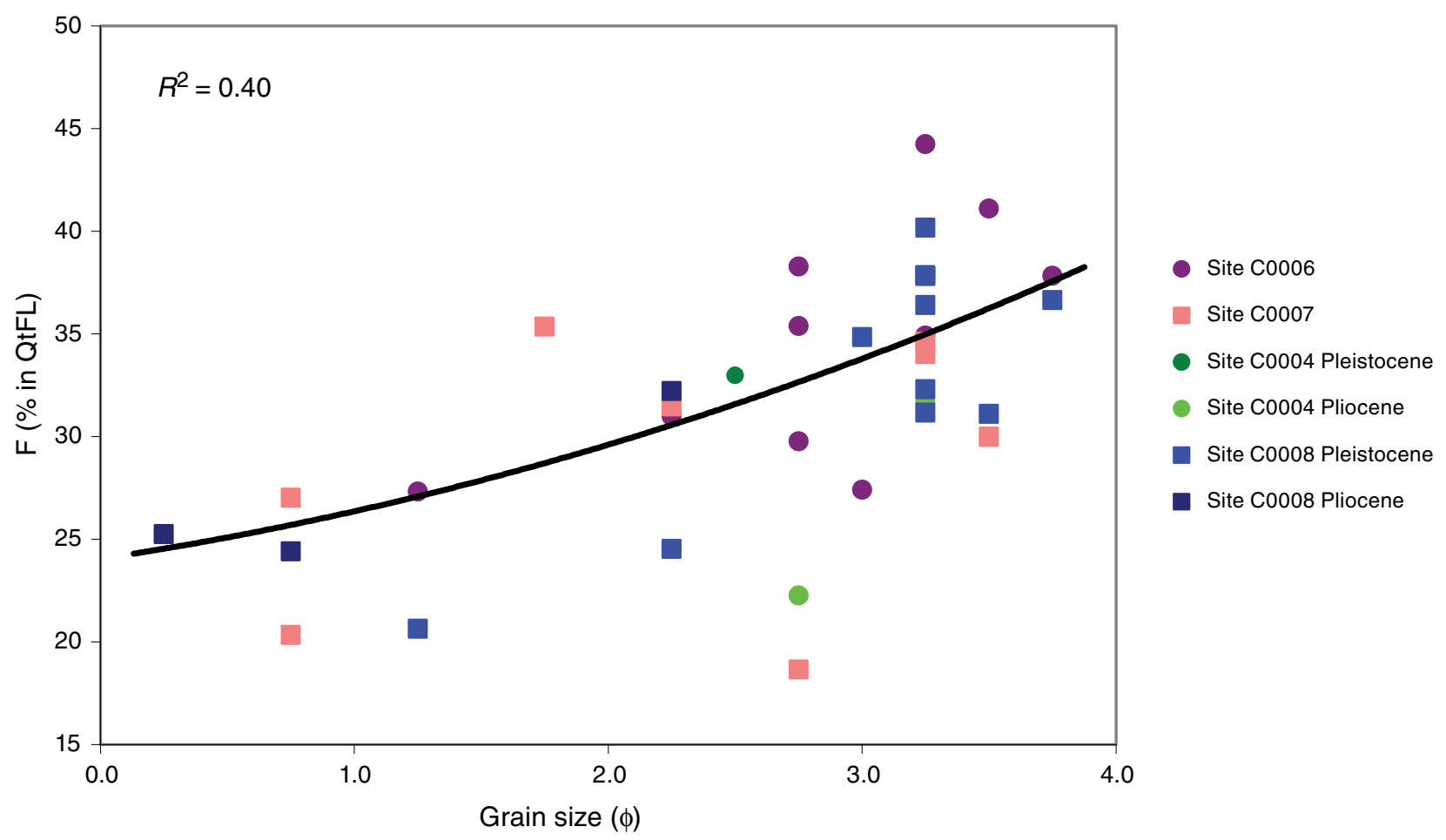


Table T1. General sample information and raw point-count data, Sites C0004, C0006, C0007, and C0008. (Continued on next two pages.)

\begin{tabular}{|c|c|c|c|c|c|c|c|c|}
\hline $\begin{array}{l}\text { Hole, core, section, } \\
\text { interval }(\mathrm{cm})\end{array}$ & Unit & $\begin{array}{c}\text { Age } \\
\text { (nannofossil zone) }\end{array}$ & $\begin{array}{l}\text { Depth } \\
\text { (mbsf) }\end{array}$ & Facies & $\begin{array}{l}\text { Grain size } \\
(\phi)\end{array}$ & $\begin{array}{l}\text { Monocrystalline } \\
\text { quartz }\end{array}$ & $\begin{array}{l}\text { Polycrystalline } \\
\text { quartz }\end{array}$ & $\begin{array}{l}\text { Monocrystalline } \\
\text { Ca-plagioclase }\end{array}$ \\
\hline 316-C0006E-5H-2, 47.5-48.5 & IIA & NN19 & 35.1 & Accreted trench wedge & 2.75 & 83 & 0 & 147 \\
\hline 316-C0006E-7H-5, 46-48 & IIA & NN19 & 43.6 & Accreted trench wedge & 3.00 & 120 & 3 & 122 \\
\hline 316-C0006E-11H-3, 45-47 & IIA & NN19 & 67.4 & Accreted trench wedge & 3.50 & 102 & 1 & 172 \\
\hline 316-C0006E-11H-6, 45-47 & IIA & NN19 & 69.4 & Accreted trench wedge & 3.25 & 132 & 2 & 154 \\
\hline 316-C0006E-11H-7, 45-47 & IIA & NN19 & 70.8 & Accreted trench wedge & 3.25 & 93 & 1 & 182 \\
\hline 316-C0006E-16X-CC, 10-12 & IIB & NN19 younger & 90.81 & Accreted trench wedge & 2.75 & 95 & 2 & 160 \\
\hline 316-C0006E-19X-2, 10-12 & IIB & NN19 younger & 117 & Accreted trench wedge & 2.75 & 73 & 3 & 126 \\
\hline 316-C0006E-22X-6, 105-107 & IIB & NN19 younger & 151.1 & Accreted trench wedge & 3.75 & 91 & 5 & 147 \\
\hline 316-C0006E-26X-2, 109-111 & IIC & NN19 younger & 184.9 & Accreted trench wedge & 1.25 & 146 & 1 & 116 \\
\hline 316-C0006E-31X-1, 120-122 & IIC & NN19 older & 231 & Accreted trench wedge & 2.25 & 88 & & 123 \\
\hline 316-C0007B-1H-6, 63-65 & I & NN19 & 2.1 & Slope apron & 3.25 & 110 & 7 & 141 \\
\hline 316-C0007C-1H-7, 13-15 & 1 & NN19 & 18.5 & Slope apron & 3.25 & 112 & 4 & 150 \\
\hline 316-C0007C-7X-4, 136-137 & IIA & NN19 & 66.3 & Accreted trench wedge & 3.50 & 103 & & 130 \\
\hline 316-C0007C-12X-1, 24-26 & IIA & NN19 & 109.9 & Accreted trench wedge & 0.75 & 153 & 10 & 122 \\
\hline $316-\mathrm{C} 0007 \mathrm{C}-16 \mathrm{H}-1,81-83$ & IIA & NN19 reversal & 148.4 & Accreted trench wedge & 1.75 & 110 & 7 & 159 \\
\hline $316-\mathrm{C} 0007 \mathrm{C}-17 \mathrm{H}-1,13-15$ & IIA & NN? & 157.2 & Accreted trench wedge & 2.25 & 68 & 12 & 137 \\
\hline 316-C0007D-12R-1, 16-18 & IIA & NN19 & 275.7 & Accreted trench wedge & 0.75 & 119 & 14 & 96 \\
\hline 316-C0007D-35R-1, 16-18 & III & Pleistocene? & 484.15 & subducted trench wedge & 2.75 & 102 & 5 & 73 \\
\hline $316-\mathrm{C} 0004 \mathrm{C}-5 \mathrm{H}-8,10-20$ & 1 & NN19 & 43.8 & Slope apron & 2.50 & 66 & 3 & 153 \\
\hline 316-C0004C-10H-1, 17-20 & IIA & NN16 & 81.04 & Mass transport complex & 3.25 & 91 & 5 & 130 \\
\hline 316-C0004C-12X-CC, 86-88 & IIA & NN16 & 99.4 & Mass transport complex & 2.75 & 115 & 11 & 103 \\
\hline 316-C0008A-6H-1, 14-16 & IA & NN19 & 44.2 & Slope basin & 3.25 & 77 & 2 & 165 \\
\hline 316-C0008C-6H-5, 92-95 & IA & NN19 & 49 & Slope basin & 3.25 & 120 & 9 & 161 \\
\hline 316-C0008A-7H-7, 107-109 & IA & NN19 & 61.4 & Slope basin & 1.25 & 113 & 1 & 78 \\
\hline 316-C0008C-9H-3, 82-84 & IA & NN19 & 70.1 & Slope basin & 3.25 & 89 & 3 & 168 \\
\hline $316-\mathrm{C} 0008 \mathrm{C}-10 \mathrm{H}-7,15-17$ & IA & NN19 & 81.7 & Slope basin & 2.25 & 80 & 1 & 103 \\
\hline 316-C0008A-11H-6, 83-85 & IA & NN19 & 97 & Slope basin & 3 & 152 & 8 & 157 \\
\hline 316-C0008C-11H-11, 89-90 & IA & NN19 & 89.2 & Slope basin & 3.25 & 91 & 3 & 162 \\
\hline 316-C0008A-13H-6, 110-112 & IA & NN19 & 116.2 & Slope basin & 3.5 & 137 & 6 & 133 \\
\hline 316-C0008A-23H-8, 7-9 & IA & NN19 & 185.3 & Slope basin & 3.75 & 128 & 2 & 161 \\
\hline 316-C0008A-24H-3, 110-112 & IA & NN19 & 190.1 & Slope basin & 3.25 & 84 & 2 & 144 \\
\hline 316-C0008A-26H-1, 118-120 & IA & NN19 & 202.1 & Slope basin & 3.25 & 126 & 4 & 142 \\
\hline 316-C0008A-34X-1, 44-45 & ॥ & NN17 & 272.7 & Accreted trench wedge & 0.25 & 76 & 1 & 93 \\
\hline 316-C0008A-35X-CC, 19-20 & II & NN16 & 282.2 & Accreted trench wedge & 0.75 & 70 & 6 & 98 \\
\hline 316-C0008A-36X-1, 18-19 & II & NN16 & 291.4 & Accreted trench wedge & 2.25 & 56 & 4 & 134 \\
\hline
\end{tabular}

$\mathrm{MRF}=$ metamorphic lithic (rock) fragments. 


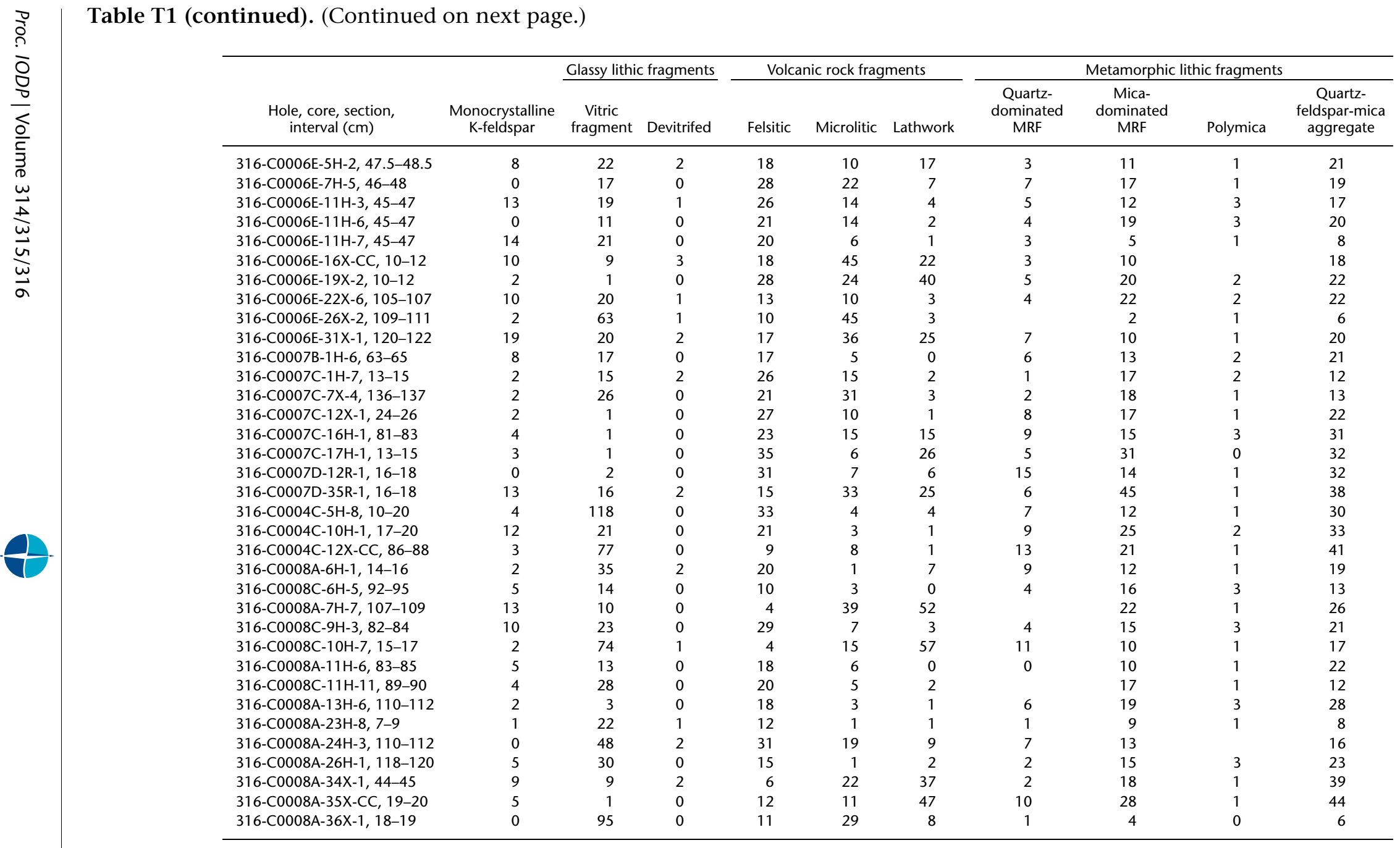




\begin{tabular}{|c|c|c|c|c|c|c|c|c|c|c|c|}
\hline & \multirow[b]{2}{*}{$\begin{array}{l}\text { Hole, core, section, } \\
\text { interval }(\mathrm{cm})\end{array}$} & \multicolumn{4}{|c|}{ Sedimentary lithic fragments } & \multicolumn{5}{|c|}{ Non-QtFL grains } & \multirow[b]{2}{*}{$\begin{array}{l}\text { Total } \\
\text { points }\end{array}$} \\
\hline & & Chert & $\begin{array}{l}\text { Cherty } \\
\text { argillite }\end{array}$ & Mudstone & Siltstone & Other & $\begin{array}{l}\text { Heavy } \\
\text { minerals }\end{array}$ & Micas & $\begin{array}{c}\text { Carbonate } \\
\text { bioclast }\end{array}$ & $\begin{array}{l}\text { Silicate } \\
\text { bioclast }\end{array}$ & \\
\hline & 316-C0006E-5H-2, 47.5-48.5 & 11 & 6 & 77 & 1 & 9 & 42 & 4 & 0 & 0 & 493 \\
\hline & 316-C0006E-7H-5, 46-48 & 28 & 4 & 45 & 5 & 7 & 37 & 6 & 5 & 0 & 500 \\
\hline & $316-\mathrm{C} 0006 \mathrm{E}-11 \mathrm{H}-3,45-47$ & 15 & 5 & 41 & & 2 & 24 & 23 & 3 & 0 & 502 \\
\hline & 316-C0006E-11H-6, 45-47 & 12 & 4 & 41 & 2 & 4 & 28 & 22 & 4 & 0 & 499 \\
\hline & 316-C0006E-11H-7, 45-47 & 18 & 3 & 64 & 3 & 6 & 19 & 30 & 2 & 0 & 500 \\
\hline & 316-C0006E-16X-CC, 10-12 & 13 & 5 & 28 & 3 & 6 & 41 & 5 & 5 & 1 & 502 \\
\hline & 316-C0006E-19X-2, 10-12 & 17 & 3 & 54 & 10 & 4 & 59 & 9 & 0 & 0 & 503 \\
\hline & 316-C0006E-22X-6, 105-107 & 11 & 4 & 45 & 5 & 5 & 51 & 25 & 3 & 1 & 500 \\
\hline & 316-C0006E-26X-2, 109-111 & 14 & 1 & 19 & 2 & 3 & 32 & 31 & 2 & 0 & 500 \\
\hline & 316-C0006E-31X-1, 120-122 & 31 & 7 & 45 & 7 & 4 & 34 & 3 & 3 & 0 & 502 \\
\hline & 316-C0007B-1H-6, 63-65 & 18 & 3 & 65 & 5 & 7 & 29 & 20 & 3 & 3 & 500 \\
\hline & $316-\mathrm{C} 0007 \mathrm{C}-1 \mathrm{H}-7,13-15$ & 23 & 2 & 53 & 1 & 6 & 34 & 16 & 6 & 1 & 502 \\
\hline & 316-C0007C-7X-4, 136-137 & 14 & 6 & 68 & 2 & 8 & 29 & 20 & 5 & 0 & 502 \\
\hline & $316-C 0007 C-12 X-1,24-26$ & 34 & 7 & 39 & 5 & 5 & 14 & 21 & 1 & 0 & 500 \\
\hline & $316-\mathrm{C} 0007 \mathrm{C}-16 \mathrm{H}-1,81-83$ & 29 & 5 & 31 & 4 & 7 & 22 & 13 & 1 & 0 & 504 \\
\hline & $316-\mathrm{C} 0007 \mathrm{C}-17 \mathrm{H}-1,13-15$ & 13 & 2 & 62 & 12 & 6 & 40 & 7 & 3 & 0 & 501 \\
\hline & 316-C0007D-12R-1, 16-18 & 54 & 14 & 59 & 8 & 6 & 12 & 9 & 3 & 0 & 502 \\
\hline & 316-C0007D-35R-1, 16-18 & 24 & 4 & 52 & 7 & 5 & 31 & 4 & 3 & 0 & 504 \\
\hline & $316-\mathrm{C} 0004 \mathrm{C}-5 \mathrm{H}-8,10-20$ & 4 & 1 & 36 & 0 & 1 & 11 & 10 & 1 & 1 & 500 \\
\hline & $316-\mathrm{C} 0004 \mathrm{C}-10 \mathrm{H}-1,17-20$ & 36 & 7 & 47 & 5 & 6 & 39 & 9 & 1 & 0 & 503 \\
\hline & $316-\mathrm{C} 0004 \mathrm{C}-12 \mathrm{X}-\mathrm{CC}, 86-88$ & 11 & 12 & 45 & 5 & 7 & 9 & 6 & 1 & 1 & 500 \\
\hline & $316-\mathrm{C} 0008 \mathrm{~A}-6 \mathrm{H}-1,14-16$ & 15 & 8 & 60 & 6 & 4 & 38 & 11 & 6 & 0 & 500 \\
\hline & $316-\mathrm{C} 0008 \mathrm{C}-6 \mathrm{H}-5,92-95$ & 34 & 3 & 59 & 2 & 4 & 24 & 11 & 3 & 2 & 500 \\
\hline & 316-C0008A-7H-7, 107-109 & 22 & 7 & 42 & 11 & 5 & 49 & 5 & & 1 & 501 \\
\hline & $316-\mathrm{C} 0008 \mathrm{C}-9 \mathrm{H}-3,82-84$ & 17 & 5 & 43 & 3 & 3 & 19 & 20 & 8 & 7 & 500 \\
\hline & $316-\mathrm{C} 0008 \mathrm{C}-10 \mathrm{H}-7,15-17$ & 32 & 2 & 18 & & 1 & 18 & 36 & 9 & 8 & 500 \\
\hline & 316-C0008A-11H-6, 83-85 & 16 & 5 & 50 & 2 & 4 & 21 & 13 & 1 & 0 & 504 \\
\hline & 316-C0008C-11H-11, 89-90 & 25 & 4 & 61 & 4 & 5 & 31 & 11 & 10 & 4 & 500 \\
\hline & $316-C 0008 \mathrm{~A}-13 \mathrm{H}-6,110-112$ & 26 & 7 & 37 & 5 & 6 & 22 & 26 & 1 & 3 & 492 \\
\hline & $316-\mathrm{C} 0008 \mathrm{~A}-23 \mathrm{H}-8,7-9$ & 17 & 6 & 71 & & 3 & 31 & 14 & 4 & 6 & 500 \\
\hline & $316-\mathrm{C} 0008 \mathrm{~A}-24 \mathrm{H}-3,110-112$ & 20 & 6 & 56 & 5 & 4 & 24 & 10 & 0 & 0 & 500 \\
\hline & $316-\mathrm{C} 0008 \mathrm{~A}-26 \mathrm{H}-1,118-120$ & 24 & 6 & 53 & 4 & 7 & 26 & 11 & 2 & 1 & 502 \\
\hline & 316-C0008A-34X-1, 44-45 & 33 & 7 & 46 & 3 & 2 & 91 & 3 & 1 & 6 & 507 \\
\hline & $316-\mathrm{C} 0008 \mathrm{~A}-35 \mathrm{X}-\mathrm{CC}, 19-20$ & 26 & 6 & 49 & 8 & 6 & 68 & 6 & 1 & 0 & 503 \\
\hline & 316-C0008A-36X-1, 18-19 & 10 & 4 & 51 & 3 & 6 & 54 & 9 & 14 & 0 & 499 \\
\hline
\end{tabular}


Table T2. Calculated parameters for detrital modes, Sites C0004, C0006, C0007, and C0008. (Continued on next page.)

\begin{tabular}{|c|c|c|c|c|c|c|c|c|c|}
\hline $\begin{array}{l}\text { Hole, core, section, } \\
\text { interval }(\mathrm{cm})\end{array}$ & Facies & $\begin{array}{l}\text { Grain size } \\
\qquad(\phi)\end{array}$ & Qt & $\mathrm{F}$ & L & Qm & $P$ & K & $F /(Q+F)$ \\
\hline 316-C0006E-5H-2, 47.5-48.5 & Accreted trench wedge & 2.75 & 18.9 & 35.4 & 45.7 & 34.9 & 61.8 & 3.4 & 0.65 \\
\hline 316-C0006E-7H-5, 46-48 & Accreted trench wedge & 3.00 & 27.6 & 27.4 & 44.9 & 49.6 & 50.4 & 0.0 & 0.50 \\
\hline $316-C 0006 \mathrm{E}-11 \mathrm{H}-3,45-47$ & Accreted trench wedge & 3.50 & 22.9 & 41.1 & 36.0 & 35.5 & 59.9 & 4.5 & 0.64 \\
\hline 316-C0006E-11H-6, 45-47 & Accreted trench wedge & 3.25 & 30.4 & 34.9 & 34.7 & 46.2 & 53.8 & 0.0 & 0.53 \\
\hline 316-C0006E-11H-7, 45-47 & Accreted trench wedge & 3.25 & 21.2 & 44.2 & 34.5 & 32.2 & 63.0 & 4.8 & 0.68 \\
\hline 316-C0006E-16X-CC, 10-12 & Accreted trench wedge & 2.75 & 21.8 & 38.3 & 39.9 & 35.8 & 60.4 & 3.8 & 0.64 \\
\hline 316-C0006E-19X-2, 10-12 & Accreted trench wedge & 2.75 & 17.7 & 29.8 & 52.6 & 36.3 & 62.7 & 1.0 & 0.63 \\
\hline 316-C0006E-22X-6, 105-107 & Accreted trench wedge & 3.75 & 23.1 & 37.8 & 39.0 & 36.7 & 59.3 & 4.0 & 0.62 \\
\hline 316-C0006E-26X-2, 109-111 & Accreted trench wedge & 1.25 & 34.0 & 27.3 & 38.7 & 55.3 & 43.9 & 0.8 & 0.45 \\
\hline \multirow[t]{2}{*}{ 316-C0006E-31X-1, 120-122 } & Accreted trench wedge & 2.25 & 19.2 & 31.0 & 49.8 & 38.3 & 53.5 & 8.3 & 0.62 \\
\hline & Average: & 2.85 & 23.7 & 34.7 & 41.6 & 40.1 & 56.9 & 3.1 & 0.59 \\
\hline 316-C0007B-1H-6, 63-65 & Slope apron & 3.25 & 26.7 & 34.0 & 39.3 & 42.5 & 54.4 & 3.1 & 0.56 \\
\hline \multirow{2}{*}{$316-C 0007 \mathrm{C}-1 \mathrm{H}-7,13-15$} & Slope apron & 3.25 & 26.4 & 34.6 & 39.0 & 42.4 & 56.8 & 0.8 & 0.57 \\
\hline & Average: & 3.25 & 26.6 & 34.3 & 39.1 & 42.4 & 55.6 & 1.9 & 0.6 \\
\hline 316-C0007C-7X-4, 136-137 & Accreted trench wedge & 3.50 & 23.4 & 30.0 & 46.6 & 43.8 & 55.3 & 0.9 & 0.56 \\
\hline 316-C0007C-12X-1, 24-26 & Accreted trench wedge & 0.75 & 35.5 & 27.0 & 37.5 & 55.2 & 44.0 & 0.7 & 0.43 \\
\hline 316-C0007C-16H-1, 81-83 & Accreted trench wedge & 1.75 & 25.4 & 35.4 & 39.3 & 40.3 & 58.2 & 1.5 & 0.58 \\
\hline $316-\mathrm{C} 0007 \mathrm{C}-17 \mathrm{H}-1,13-15$ & Accreted trench wedge & 2.25 & 18.0 & 31.5 & 50.6 & 32.7 & 65.9 & 1.4 & 0.64 \\
\hline \multirow[t]{2}{*}{ 316-C0007D-12R-1, 16-18 } & Accreted trench wedge & 0.75 & 28.2 & 20.3 & 51.5 & 55.3 & 44.7 & 0.0 & 0.42 \\
\hline & $\begin{array}{l}\text { Average: }\end{array}$ & 1.80 & 26.09 & 28.83 & 45.07 & 45.48 & 53.62 & 0.90 & 0.53 \\
\hline 316-C0007D-35R-1, 16-18 & Subducted trench wedge & 2.75 & 23.2 & 18.7 & 58.1 & 54.3 & 38.8 & 6.9 & 0.45 \\
\hline $316-\mathrm{C} 0004 \mathrm{C}-5 \mathrm{H}-8,10-20$ & Slope apron & 2.50 & 14.5 & 33.0 & 52.5 & 29.6 & 68.6 & 1.8 & 0.69 \\
\hline 316-C0004C-10H-1, 17-20 & Mass transport complex & 3.25 & 21.4 & 31.7 & 46.9 & 39.1 & 55.8 & 5.2 & 0.60 \\
\hline \multirow[t]{2}{*}{ 316-C0004C-12X-CC, 86-88 } & Mass transport complex & 2.75 & 26.5 & 22.3 & 51.3 & 52.0 & 46.6 & 1.4 & 0.46 \\
\hline & Average: & 3.00 & 23.9 & 27.0 & 49.1 & 45.5 & 51.2 & 3.3 & 0.53 \\
\hline 316-C0008A-6H-1, 14-16 & Slope basin & 3.25 & 17.9 & 37.9 & 44.2 & 31.6 & 67.6 & 0.8 & 0.68 \\
\hline $316-\mathrm{C} 0008 \mathrm{C}-6 \mathrm{H}-5,92-95$ & Slope basin & 3.25 & 28.3 & a36.4 & 35.3 & 42.0 & 56.3 & 1.7 & 0.56 \\
\hline 316-C0008A-7H-7, 107-109 & Slope basin & 1.25 & 25.9 & 20.6 & 53.5 & 55.4 & 38.2 & 6.4 & 0.44 \\
\hline 316-C0008C-9H-3, 82-84 & Slope basin & 3.25 & 20.8 & 40.2 & 39.1 & 33.3 & 62.9 & 3.7 & 0.66 \\
\hline $316-\mathrm{C} 0008 \mathrm{C}-10 \mathrm{H}-7,15-17$ & Slope basin & 2.25 & 18.9 & 24.5 & 56.5 & 43.2 & 55.7 & 1.1 & 0.56 \\
\hline 316-C0008A-11H-6, 83-85 & Slope basin & 3.00 & 34.4 & 34.8 & 30.8 & 48.4 & 50.0 & 1.6 & 0.50 \\
\hline 316-C0008C-11H-11, 89-90 & Slope basin & 3.25 & 21.4 & 37.8 & 40.8 & 35.4 & 63.0 & 1.6 & 0.64 \\
\hline 316-C0008A-13H-6, 110-112 & Slope basin & 3.50 & 32.9 & 31.1 & 35.9 & 50.4 & 48.9 & 0.7 & 0.49 \\
\hline 316-C0008A-23H-8, 7-9 & Slope basin & 3.75 & 29.4 & 36.7 & 33.9 & 44.1 & 55.5 & 0.3 & 0.55 \\
\hline $316-\mathrm{C} 0008 \mathrm{~A}-24 \mathrm{H}-3,110-112$ & Slope basin & 3.25 & 18.6 & 31.2 & 50.2 & 36.8 & 63.2 & 0.0 & 0.63 \\
\hline \multirow[t]{2}{*}{ 316-C0008A-26H-1, 118-120 } & Slope basin & 3.25 & 28.6 & 32.3 & 39.1 & 46.2 & 52.0 & 1.8 & 0.53 \\
\hline & Average: & 3.02 & 25.2 & 33.0 & 41.8 & 42.4 & 55.8 & 1.8 & 0.57 \\
\hline 316-C0008A-34X, 44-45 & Accreted trench wedge & 0.25 & 19.1 & 25.2 & 55.7 & 42.7 & 52.2 & 5.1 & 0.57 \\
\hline 316-C0008A-35X-CC, 19-20 & Accreted trench wedge & 0.75 & 18.0 & 24.4 & 57.6 & 40.5 & 56.6 & 2.9 & 0.58 \\
\hline \multirow[t]{2}{*}{ 316-C0008A-36X-1, 18-19 } & Accreted trench wedge & 2.25 & 14.4 & 32.2 & 53.4 & 29.5 & 70.5 & 0.0 & 0.69 \\
\hline & $\begin{array}{l}\text { Average: } \\
\text { A }\end{array}$ & 1.08 & 17.2 & 27.3 & 55.5 & 37.5 & 59.8 & 2.6 & 0.61 \\
\hline
\end{tabular}

Grain parameters: $\mathrm{Qt}=$ quartz in normalized $\mathrm{QtFL}, \mathrm{F}=$ feldspar in normalized $\mathrm{QtFL}, \mathrm{L}=$ lithic fragments in normalized $\mathrm{QtFL}, \mathrm{Qm}=\mathrm{monocrystal}-$ line quartz in normalized QmPK, P = plagioclase in normalized QmPK, K = K-feldspar in normalized QmPk, F/(Q + F) $=$ feldspar/(detrital quartz + feldspar), $P /(P+K)=$ plagioclase/total feldspar, $L s=$ sedimentary lithics in normalized total lithics, $L m=$ metamorphic lithics in normalized total lithics, $L v=$ volcanic lithics in normalized total lithics, Felsitic = volcanic lithic with felsitic fabric, Microlitic = volcanic lithic with microlitic fabric, Lathwork = volcanic lithic with lathwork fabric. 
Table T2 (continued).

\begin{tabular}{|c|c|c|c|c|c|c|c|c|}
\hline $\begin{array}{l}\text { Hole, core, section, } \\
\text { interval }(\mathrm{cm})\end{array}$ & Facies & $P /(P+K)$ & Ls & $\mathrm{Lm}$ & Lv & Felsitic & Microlitic & Lathwork \\
\hline 316-C0006E-5H-2, 47.5-48.5 & Accreted trench wedge & 0.95 & 47.5 & 18.0 & 34.5 & 40.0 & 22.2 & 37.8 \\
\hline 316-C0006E-7H-5, 46-48 & Accreted trench wedge & 1.00 & 41.0 & 22.0 & 37.0 & 49.1 & 38.6 & 12.3 \\
\hline $316-C 0006 \mathrm{E}-11 \mathrm{H}-3,45-47$ & Accreted trench wedge & 0.93 & 37.7 & 22.8 & 39.5 & 59.1 & 31.8 & 9.1 \\
\hline 316-C0006E-11H-6, 45-47 & Accreted trench wedge & 1.00 & 38.6 & 30.1 & 31.4 & 56.8 & 37.8 & 5.4 \\
\hline 316-C0006E-11H-7, 45-47 & Accreted trench wedge & 0.93 & 57.5 & 11.1 & 31.4 & 74.1 & 22.2 & 3.7 \\
\hline 316-C0006E-16X-CC, 10-12 & Accreted trench wedge & 0.94 & 27.7 & 17.5 & 54.8 & 21.2 & 52.9 & 25.9 \\
\hline 316-C0006E-19X-2, 10-12 & Accreted trench wedge & 0.98 & 37.2 & 21.7 & 41.2 & 30.4 & 26.1 & 43.5 \\
\hline 316-C0006E-22X-6, 105-107 & Accreted trench wedge & 0.94 & 40.1 & 30.9 & 29.0 & 50.0 & 38.5 & 11.5 \\
\hline 316-C0006E-26X-2, 109-111 & Accreted trench wedge & 0.98 & 21.6 & 5.4 & 73.1 & 17.2 & 77.6 & 5.2 \\
\hline \multirow[t]{2}{*}{ 316-C0006E-31X-1, 120-122 } & Accreted trench wedge & 0.87 & 39.5 & 16.7 & 43.9 & 21.8 & 46.2 & 32.1 \\
\hline & Average: & 0.95 & 38.8 & 19.6 & 41.6 & 42.0 & 39.4 & 18.6 \\
\hline 316-C0007B-1H-6, 63-65 & Slope apron & 0.95 & 52.9 & 24.4 & 22.7 & 77.3 & 22.7 & 0.0 \\
\hline \multirow[t]{2}{*}{ 316-C0007C-1H-7, 13-15 } & Slope apron & 0.99 & 46.2 & 18.7 & 35.1 & 60.5 & 34.9 & 4.7 \\
\hline & Average: & 1.0 & 49.6 & 21.6 & 28.9 & 68.9 & 28.8 & 2.3 \\
\hline 316-C0007C-7X-4, 136-137 & Accreted trench wedge & 0.98 & 43.9 & 16.6 & 39.5 & 38.2 & 56.4 & 5.5 \\
\hline 316-C0007C-12X-1, 24-26 & Accreted trench wedge & 0.98 & 49.4 & 27.9 & 22.7 & 71.1 & 26.3 & 2.6 \\
\hline $316-\mathrm{C} 0007 \mathrm{C}-16 \mathrm{H}-1,81-83$ & Accreted trench wedge & 0.98 & 38.1 & 32.0 & 29.8 & 43.4 & 28.3 & 28.3 \\
\hline 316-C0007C-17H-1, 13-15 & Accreted trench wedge & 0.98 & 39.6 & 30.2 & 30.2 & 52.2 & 9.0 & 38.8 \\
\hline \multirow[t]{2}{*}{ 316-C0007D-12R-1, 16-18 } & Accreted trench wedge & 1.00 & 55.6 & 25.5 & 18.9 & 70.5 & 15.9 & 13.6 \\
\hline & Average: & 0.98 & 45.31 & 26.45 & 28.23 & 55.06 & 27.17 & 17.77 \\
\hline 316-C0007D-35R-1, 16-18 & Subducted trench wedge & 0.85 & 32.5 & 33.6 & 34.0 & 20.5 & 45.2 & 34.2 \\
\hline $316-\mathrm{C} 0004 \mathrm{C}-5 \mathrm{H}-8,10-20$ & Slope apron & 0.97 & 16.4 & 20.0 & 63.6 & 80.5 & 9.8 & 9.8 \\
\hline 316-C0004C-10H-1, 17-20 & Mass transport complex & 0.92 & 45.2 & 32.9 & 21.9 & 84.0 & 12.0 & 4.0 \\
\hline \multirow[t]{2}{*}{ 316-C0004C-12X-CC, 86-88 } & Mass transport complex & 0.97 & 29.9 & 31.1 & 38.9 & 50.0 & 44.4 & 5.6 \\
\hline & Average: & 0.94 & 37.6 & 32.0 & 30.4 & 67.0 & 28.2 & 4.8 \\
\hline 316-C0008A-6H-1, 14-16 & Slope basin & 0.99 & 45.6 & 21.0 & 33.3 & 71.4 & 3.6 & 25.0 \\
\hline $316-\mathrm{C} 0008 \mathrm{C}-6 \mathrm{H}-5,92-95$ & Slope basin & 0.97 & 60.9 & 22.4 & 16.8 & 76.9 & 23.1 & 0.0 \\
\hline 316-С0008A-7H-7, 107-109 & Slope basin & 0.86 & 34.7 & 20.8 & 44.5 & 4.2 & 41.1 & 54.7 \\
\hline $316-\mathrm{C} 0008 \mathrm{C}-9 \mathrm{H}-3,82-84$ & Slope basin & 0.94 & 39.3 & 24.9 & 35.8 & 74.4 & 17.9 & 7.7 \\
\hline $316-\mathrm{C} 0008 \mathrm{C}-10 \mathrm{H}-7,15-17$ & Slope basin & 0.98 & 21.5 & 16.1 & 62.4 & 5.3 & 19.7 & 75.0 \\
\hline 316-C0008A-11H-6, 83-85 & Slope basin & 0.97 & 51.0 & 23.1 & 25.9 & 75.0 & 25.0 & 0.0 \\
\hline 316-C0008C-11H-11, 89-90 & Slope basin & 0.98 & 52.5 & 16.8 & 30.7 & 74.1 & 18.5 & 7.4 \\
\hline 316-C0008A-13H-6, 110-112 & Slope basin & 0.99 & 48.1 & 35.9 & 16.0 & 81.8 & 13.6 & 4.5 \\
\hline 316-C0008A-23H-8, 7-9 & Slope basin & 0.99 & 62.7 & 12.7 & 24.7 & 85.7 & 7.1 & 7.1 \\
\hline 316-C0008A-24H-3, 110-112 & Slope basin & 1.00 & 37.5 & 15.5 & 47.0 & 52.5 & 32.2 & 15.3 \\
\hline \multirow[t]{2}{*}{ 316-C0008A-26H-1, 118-120 } & Slope basin & 0.97 & 48.9 & 24.2 & 27.0 & 83.3 & 5.6 & 11.1 \\
\hline & Average: & 0.97 & 45.7 & 21.2 & 33.1 & 62.2 & 18.9 & 18.9 \\
\hline 316-C0008A-34X, 44-45 & Accreted trench wedge & 0.91 & 39.6 & 26.7 & 33.8 & 9.2 & 33.8 & 56.9 \\
\hline 316-C0008A-35X-CC, 19-20 & Accreted trench wedge & 0.95 & 36.6 & 34.2 & 29.2 & 17.1 & 15.7 & 67.1 \\
\hline \multirow[t]{2}{*}{ 316-C0008A-36X-1, 18-19 } & Accreted trench wedge & 1.00 & 30.6 & 5.0 & 64.4 & 22.9 & 60.4 & 16.7 \\
\hline & Average: & 0.96 & 35.6 & 21.9 & 42.5 & 16.4 & 36.7 & 46.9 \\
\hline
\end{tabular}

